\title{
Theoretical models of the planetary nebula populations in galaxies: The ISM oxygen abundance when star formation stops $^{\star, \star \star, \star \star \star}$
}

\author{
M.G. Richer ${ }^{1}$, M.L. McCall ${ }^{2}$, and N. Arimoto ${ }^{3}$ \\ 1 DAEC, Observatoire de Meudon, F-92195 Meudon Cedex, France \\ 2 Department of Physics \& Astronomy, York University, 4700 Keele Street, North York, Ontario, Canada M3J 1P3 \\ 3 Institute of Astronomy, University of Tokyo, Mitaka, Tokyo 181, Japan
}

Received July 31, 1995; accepted July 2, 1996

\begin{abstract}
Using theoretical models of the planetary nebula populations in galaxies, we investigate whether the current oxygen abundances in bright planetary nebulae can be used to predict the oxygen abundance in the interstellar medium when star formation stopped. These models successfully reproduce a constant planetary nebula luminosity function (PNLF) peak luminosity, the PNLF shape in galaxies with and without star formation, and the mean densities and oxygen abundances observed in bright planetary nebulae in the Magellanic Clouds. To accomplish this, we had to couple the evolution of the nebular shell and the central star, and impose a massdependent nebular covering factor. In all galaxies, these models predict that a gap develops between the abundances observed in bright planetary nebulae and those in the interstellar medium when star formation stopped. This abundance gap depends primarily upon the oxygen abundance achieved in the interstellar medium when star formation stopped, though it also has some sensitivity to the history of star formation. The abundance gap is always less than 0.35 dex in these models. For the Milky Way, the predicted abundance gap, $0.14 \mathrm{dex}$, is identical to that observed. We also re-examined spectroscopic observations of planetary nebulae in diffuse elliptical galaxies to determine their interstellar medium oxygen abundances when
\end{abstract}

Send offprint requests to: M.G. Richer, richer@mesunb.obspm.fr

* Observations discussed in this paper were obtained at the Multiple Mirror Telescope Observatory, a joint facility of the University of Arizona and the Smithsonian Institution.

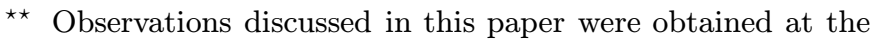
Canada-France-Hawaii Telescope, operated by the National Research Council of Canada, le Centre National de la Recherche Scientifique de France, and the University of Hawaii.

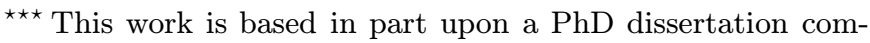
pleted by MGR at CRESS, York University, 4700 Keele Street, North York, Ontario, Canada M3J 1P3. they stopped forming stars. Allowing for the abundance gap magnifies the chemical differences between diffuse ellipticals and dwarf irregulars found by Richer \& McCall (1995). Diffuse ellipticals are confirmed to have larger oxygen abundances than similarly luminous dwarf irregulars, and to have larger $[\mathrm{O} / \mathrm{Fe}]$ ratios than dwarf irregulars with the same oxygen abundance. The simplest explanation for both of these observations is that diffuse ellipticals formed the majority of their stars on shorter time scales than dwarf irregulars, so diffuse ellipticals cannot be the faded remnants of dwarf irregulars.

Key words: galaxies: abundances, evolution, stellar content - ISM: planetary nebulae: general — stars: AGB and post-AGB

\section{Introduction}

Over the years, extragalactic planetary nebulae have been used sporadically as probes of the chemical evolution of their parent galaxies. The most extensively studied extragalactic planetary nebulae are those in the Magellanic Clouds. In both the LMC and the SMC, planetary nebulae and HII regions have similar oxygen abundances (e.g., Dopita \& Meatheringham 1991a,b). Planetary nebulae have also seen limited duty as probes of the chemical evolution of the bulge and disk of M 31. Jacoby \& Ford (1986) obtained oxygen abundances for three planetary nebulae, one of which had a radial velocity that led them to conclude that it was a disk object. Since this disk planetary nebula had an oxygen abundance similar to that in HII regions at the same radius, Jacoby \& Ford (1986) concluded that the high oxygen abundance in M 31's outer disk was attained early in its evolution. The two halo 
planetary nebulae in M 31 had very different oxygen abundances, which Jacoby \& Ford (1986) interpreted as a manifestation of the heterogeneous line strengths previously found among M 31's globular clusters (van den Bergh 1969; Huchra et al. 1982).

Richer (1993) provided the basis for using bright planetary nebulae as probes of chemical evolution in starforming galaxies. However, it is in galaxies where star formation ceased long ago that planetary nebulae present their most exciting uses as chemical evolution probes. Here, we investigate whether, in galaxies without star formation, bright planetary nebulae can be used to determine the oxygen abundance in the interstellar medium at the time when star formation stopped.

From the viewpoint of chemical evolution, the oxygen abundance in the interstellar medium when star formation ceased (henceforth, the last epoch abundance) is particularly interesting. If bright planetary nebulae were reliable probes of the last epoch abundance, many questions concerning the formation and evolution of galaxies lacking recent star formation could be investigated. Among these: What fraction of their gas did ellipticals turn into stars? How does this fraction vary with the luminosity or the total mass? Was the stellar initial mass function during the star-forming epoch similar to that observed in star-forming galaxies today? What was the time scale over which these galaxies formed? Are diffuse elliptical galaxies (spheroidals) the faded remnants of dwarf irregular galaxies? Excluding the last one, these questions could equally well be asked concerning the bulges of spiral galaxies.

Although bright planetary nebulae are excellent probes of the current oxygen abundance in the interstellar medium in the Magellanic Clouds (Richer 1993), it is not clear that they will be such effective indicators of the last epoch abundance in galaxies where star formation stopped long ago. In the Magellanic Clouds and the Milky Way, the maximum attainable [OIII] $\lambda 5007$ luminosities and $[\mathrm{OIII}] \lambda 5007 / \mathrm{H} \beta$ ratios increase as the oxygen abundance increases, but they become insensitive to changes in the oxygen abundance at high abundances (Richer \& McCall 1995, henceforth RM95). Depending upon the history of star formation, in galaxies where the oxygen abundance is higher, the bright planetary nebulae may originate from stars occupying a broader range of oxygen abundances. Were this to happen, their mean abundance would become a progressively less reliable measure of the oxygen abundance in the interstellar medium when star formation ceased. Unfortunately, it is impossible to determine directly whether planetary nebulae measure the last epoch abundance in galaxies where star formation stopped long ago. In these galaxies, the only way to investigate how the present abundances in bright planetary nebulae are related to the last epoch abundances is to build models of the planetary nebula population. Fortunately, observations cover a sufficient range of conditions in galaxies, both with and without star formation, that they can place robust constraints upon the free parameters.

Previous studies of planetary nebula luminosity functions (PNLFs; e.g., Jacoby 1989; Méndez et al. 1993) have successfully accounted for the near constancy of the peak luminosity by incorporating a narrow central star mass distribution. Such a mass distribution is reasonable given the narrow mass distribution for white dwarfs (e.g., Weidemann 1990), which are the direct descendants of planetary nebula central stars. Since these investigations sought to explain the existence and behaviour of PNLFs, primarily at the bright end, the lack of direct coupling between the central star mass distribution and the history of star formation was not a shortcoming. In order to relate the abundances in bright planetary nebulae to the last epoch abundances, however, it is critical to maintain the explicit connection between the star formation history of the host galaxy and its planetary nebula central star mass distribution. It is in this respect that the present work differs from past efforts. In our models, the planetary nebula population is generated from a self-consistent treatment of the history of star formation and chemical evolution of its parent galaxy.

Before considering models of planetary nebula populations, we review the observed properties of planetary nebula populations in galaxies to establish the characteristics the models must display (Sect. 2). Following this, we develop the models (Sect. 3). Once a suitable model is chosen, we consider its implications regarding the utility of bright planetary nebulae as probes of the last epoch oxygen abundance (Sect. 4). As an application of these results, we re-investigate whether diffuse ellipticals and dwarf irregulars may be related by evolution (Sect. 5). Finally, we present our conclusions (Sect. 6).

\section{The observed properties of the planetary nebula populations in galaxies}

\subsection{The PNLF peak luminosity}

The driving force behind the interest in PNLFs comes from the near constancy of the peak luminosity, which makes the PNLF useful as a standard candle. So far, the only property that is observed to have any effect upon the PNLF peak luminosity is the nebular oxygen abundance (Richer 1993). Both the maximum [OIII] $\lambda 5007$ luminosity and the maximum $[\mathrm{OIII}] \lambda 5007 / \mathrm{H} \beta$ ratio attained by planetary nebulae are a function of their oxygen abundance (RM95). The effect of metallicity seems to be restricted to low oxygen abundances, however, since all bright galaxies, which are presumably oxygen-rich, appear to have similar PNLF peak luminosities.

There is no evidence that the PNLF peak luminosity is sensitive to the age of the underlying stellar population. A comparison of the PNLF peak luminosities in the LMC and the bulge of M 31 is the most stringent evidence 
that young stars do not produce brighter planetary nebulae. Undoubtedly, the planetary nebula progenitors in the LMC are younger and more massive than those in the bulge of M 31, but M 31 has the higher PNLF peak luminosity (RM95). In fact, the difference in peak luminosities between M 31 and the LMC is compatible with the expected difference in oxygen abundance (RM95). Further evidence that the PNLF peak luminosity is insensitive to its progenitor population comes from the similar PNLF peak luminosities observed in the bulge of M 31 and the disk of the Milky Way (Méndez et al. 1993), and the very similar PNLF distances that are derived for galaxies of different morphological types in the same group or cluster (e.g., Ciardullo et al. 1989a; McMillan et al. 1993).

That the maximum [OIII] $\lambda 5007$ luminosity is independent of the presence of ongoing star formation is critically important, for it implies that, excluding abundance effects, the maximum [OIII] $\lambda 5007$ luminosity is constant throughout the life of all galaxies. Fundamentally, this observation constrains the way in which planetary nebulae evolve. Some mechanism must exist that prevents the young, massive planetary nebula progenitors in starforming galaxies from producing more luminous planetary nebulae than the old, low mass planetary nebula progenitors in galaxies where star formation ended long ago.

\subsection{The shape of the PNLF}

The luminosity function for the planetary nebulae in the Magellanic Clouds has been studied to fainter levels than that for any other planetary nebula population. Jacoby (1980) constructed the deepest PNLF for the Magellanic Clouds, attaining luminosities some six magnitudes below the peak. However, subsequent work has shown that a substantial number of Jacoby's (1980) planetary nebulae were, in fact, not planetary nebulae (Boroson \& Liebert 1989). Because an accurate understanding of the PNLF is crucial to constraining models of planetary nebula populations, we first re-analyze the PNLF in the Magellanic Clouds to determine what effect interlopers had upon the luminosity function Jacoby (1980) obtained.

In addition to excluding the interlopers from Jacoby's (1980) sample, we re-calibrated his original photometry to the Jacoby (1989) system. This is necessary to define the shape of the PNLF as precisely as possible. To effect this re-calibration, we used the [OIII] $\lambda 5007$ fluxes for the Jacoby (1980) objects found in the compilation in Richer (1993), but enlarged to include the photometric fluxes from Vassiliadis et al. (1992) and Jacoby \& Kaler (1993). Henceforth, this enlarged compilation will be referred to as the complete sample (Richer 1994; see also RM95 for further details).

To generate $[\mathrm{OIII}] \lambda 5007$ magnitudes for the Jacoby (1980) objects lacking photometry on the Jacoby (1989) system, we adopted the following procedure. First, the fluxes for Jacoby's (1980) planetary nebulae appearing in the complete sample were transformed to [OIII $] \lambda 5007$ magnitudes using

$m_{5007}=-2.5 \log F(5007)-13.74$

(Allen 1973; Jacoby 1989). Second, we fit these [OIII] $\lambda 5007$ magnitudes as a function of the Jacoby (1980) magnitudes. We fit the data for planetary nebulae in the LMC and the SMC separately, since the original magnitude scales were slightly different. There were 8 and 13 planetary nebulae in the SMC and LMC, respectively, to use in this transformation. The relations between the original and new magnitude systems are shown in Fig. 1. The dispersions about the fits are $0.42 \mathrm{mag}$ for the SMC and $0.34 \mathrm{mag}$ for the LMC. Finally, we used the relations shown in Fig. 1 to calculate [OIII] $\lambda 5007$ magnitudes for the Jacoby (1980) planetary nebulae that are missing from the complete sample.

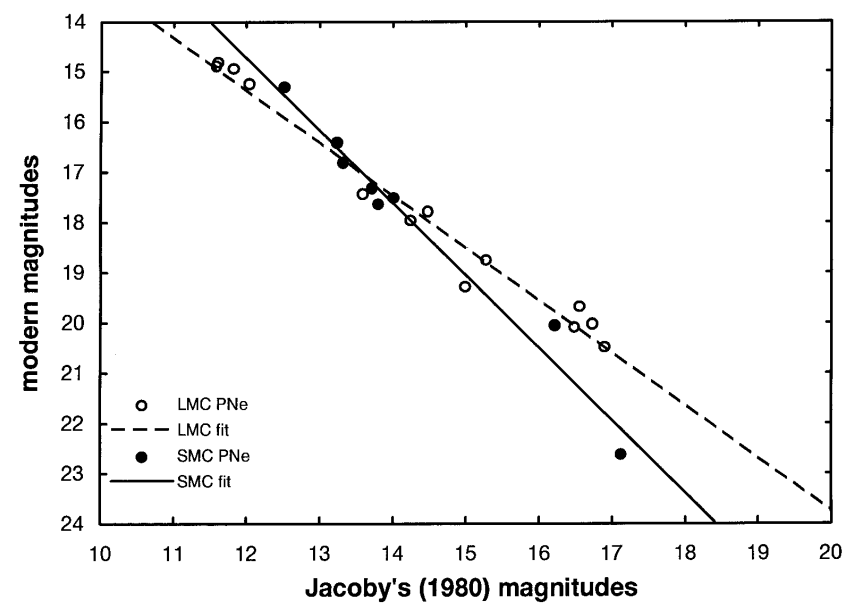

Fig. 1. The re-calibration of Jacoby's (1980) magnitude system to that defined by Eq. (1). The different symbols are defined in the legend. Although the LMC fit is effectively a zero-point shift, the slope of the SMC fit departs significantly from unity regardless of whether the faintest planetary nebula is included

Since there are only 38 planetary nebulae in the Jacoby (1980) sample, we pooled the LMC and SMC data to create a composite PNLF that was representative of the planetary nebula population in the Magellanic Clouds. Thus, for each planetary nebula in the Jacoby (1980) sample, we calculated the difference between its [OIII] $\lambda 5007 \mathrm{mag}$ nitude and the PNLF peak luminosity, adopting apparent PNLF peak magnitudes of $m_{5007}^{*}=14.35 \mathrm{mag}$ and $m_{5007}^{*}=14.93 \mathrm{mag}$ in the LMC and the SMC, respectively (Jacoby et al. 1990). Using these magnitude differences, we then computed a composite PNLF for the Magellanic Clouds that we shall refer to as the Jacoby PNLF. The Jacoby PNLF is tabulated in Col. 2 of Table 1 and shown by the heavy line in Fig. 2. (The luminosity 
functions in Table 1, like all those that follow, are cumulative luminosity functions, i.e., the fraction of all planetary nebulae exceeding a particular luminosity threshold). The Jacoby PNLF is more linear than the one Jacoby (1980) derived, especially for the first $5 \mathrm{mag}$, because many of Jacoby's (1980) fainter planetary nebula candidates were interlopers. Jacoby (1980) found that the theoretical luminosity function of Henize \& Westerlund (1963), $N\left(m_{5007}\right) \propto \mathrm{e}^{0.307 m_{5007}}$, the dashed line in Fig. 2, fit his data well. Over the 6 mag interval shown in Fig. 2, however, a chi- squared test shows that the Henize \& Westerlund (1963) luminosity function is statistically distinguishable from the Jacoby PNLF, i.e., $P\left(\chi^{2}\right)<0.01$.

Table 1. PNLFs for the Magellanic Clouds

\begin{tabular}{ccccccc}
\hline \hline Cut-Off $^{\mathrm{a}}$ & Jacoby & Number & LMC & Number & SMC & Number \\
\hline 0 & 0 & 0 & 0 & 0 & 0 & 0 \\
1 & 0.16 & 6 & 0.11 & 23 & 0.13 & 11 \\
2 & 0.24 & 9 & 0.24 & 50 & 0.24 & 20 \\
3 & 0.37 & 14 & 0.35 & 73 & 0.37 & 31 \\
4 & 0.50 & 19 & 0.46 & 98 & 0.40 & 34 \\
5 & 0.66 & 25 & 0.51 & 108 & 0.49 & 41 \\
6 & 1.00 & 38 & 0.58 & 122 & 0.50 & 42 \\
\hline
\end{tabular}

a This is the [OIII $] \lambda 5007$ luminosity threshold, in magnitudes, below the PNLF peak luminosity.

Table 1 also tabulates the global luminosity functions for the LMC and SMC based upon the complete sample. The global luminosity functions complement the Jacoby PNLF in that the bright end is much better defined on account of the complete sample's much larger areal coverage. The global luminosity functions have been normalized relative to the Jacoby PNLF by forcing the fractions of planetary nebulae within $2 \mathrm{mag}$ of the luminosity function peak to be equal. Assuming that the shape of the Jacoby PNLF is representative of the shape of the true PNLF in the Magellanic Clouds, Fig. 2 shows that the global luminosity function for the LMC is approximately complete for planetary nebulae within 4 mag of the luminosity function peak. By the same reasoning, the SMC's global luminosity function is approximately complete for planetary nebulae within $3 \mathrm{mag}$ of the luminosity function peak. As might be suspected from Fig. 2, a chi-squared test shows that, over the first $4 \mathrm{mag}$, the LMC's global luminosity function is also statistically different from the Henize \& Westerlund (1963) luminosity function. However, the number of SMC objects within the first 3 mag of its global luminosity function is too small to rule out compatibility with the Henize \& Westerlund (1963) luminosity function.

Recently, Ciardullo (personal communication; see also Ciardullo 1995) has obtained a deep luminosity function for the bulge of M 31 that extends to 4 mag below the peak luminosity. Their preliminary analysis in-

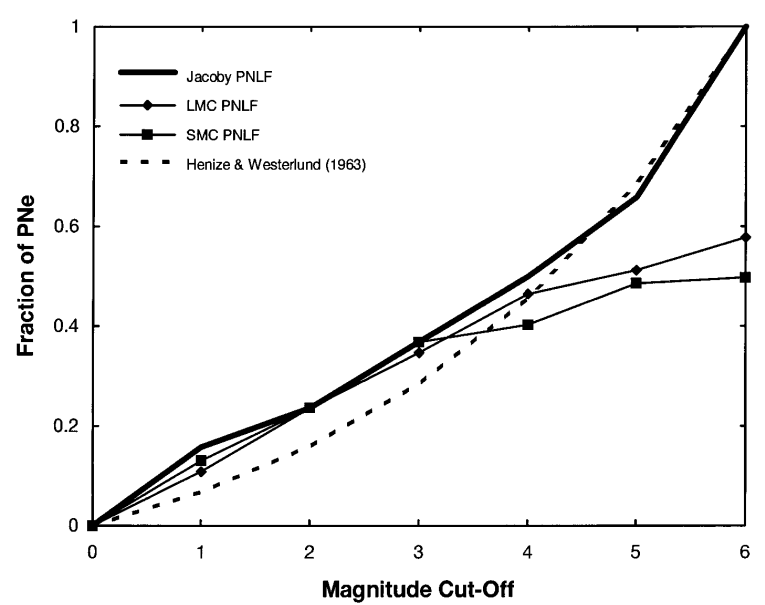

Fig. 2. The cumulative PNLF for Magellanic Cloud planetary nebulae. The Jacoby (1980) PNLF is based upon re-calibrated magnitudes for the objects not included in the complete sample (see text). The PNLFs for the LMC and SMC planetary nebulae are based upon objects in the complete sample only. Compared to the Jacoby (1980) sample, the LMC PNLF becomes seriously incomplete beyond about 4 mag below the luminosity function peak, while that for the SMC becomes incomplete beyond about 3 mag below the luminosity function peak. Both the Jaboby and LMC PNLFs are statistically different from the Henize \& Westerlund (1963) luminosity function

dicates that the PNLF in the bulge of M 31 is statistically compatible with the Henize \& Westerlund (1963) luminosity function. Thus, the shapes of the PNLFs in the LMC and the bulge of M 31 are statistically different. Indeed, using sample A from Ciardullo et al. (1989b) to define the first $2.5 \mathrm{mag}$ of PNLF in the bulge of M 31, both chi-squared and Kolmogorov-Smirnov tests indicate that M 31's PNLF differs statistically from the LMC's global PNLF. Presumably, the variation in the shape of the PNLF is a result of the different evolution of planetary nebulae derived from progenitors of different masses.

\subsection{The planetary nebula specific density}

Observations now clearly demonstrate that the specific density of bright planetary nebulae (the number per unit luminosity) depends upon the underlying stellar population. Peimbert (1990) was the first to notice that galaxies with higher luminosities or redder colours have a lower specific density of bright planetary nebulae. Further work has only reinforced these trends (Hui et al. 1993). Both trends invite suspicion that they are the result of abundance variations since larger, more luminous galaxies are also more metal-rich.

Peimbert (1990) argued that a change of the central star mass with metallicity is not responsible for the specific density trends, because galaxies in groups are observed to have different specific densities but similar 
maximum $[\mathrm{OIII}] \lambda 5007$ luminosities. Another suggestion is that bright planetary nebulae arise from a subset of the planetary nebula progenitors that produce planetary nebulae with more massive central stars, and that the fraction of progenitors that produce more massive central stars decreases as the galaxy's luminosity or metallicity increases (Peimbert 1990; Ciardullo et al. 1991).

\subsection{The oxygen abundances for bright planetary nebulae in the Magellanic Clouds}

The oxygen abundances observed in bright planetary nebulae in the Magellanic Clouds constrain planetary nebula evolution through the number of planetary nebulae arising from oxygen-rich progenitors, i.e., recent star formation. The number of such planetary nebulae depends upon the history of star formation, the initial-to-final mass relation for planetary nebula progenitors, and the evolutionary time scales for planetary nebula central stars. These three factors affect the number of planetary nebulae expected from recently formed progenitors in different ways. First, the death rate from younger stellar populations will be higher than that from older populations (e.g., Renzini \& Buzzoni 1986). Thus, per unit mass, young, metal-rich stellar populations will produce more planetary nebulae than older, more metal-poor populations, which will bias the mean oxygen abundance for bright planetary nebulae upward. Second, if the central star mass decreases significantly as the initial metallicity increases, as many stellar evolution models predict, the $[\mathrm{OIII}] \lambda 5007$ luminosities of planetary nebulae with lower oxygen abundances can be similar to those with higher abundances because the low metallicity central stars will be brighter and able to compensate for their nebula's lower [OIII] $\lambda 5007$ emission efficiency. This would cause a great deal of population mixing among bright planetary nebulae, lowering their mean abundance. Third, all evolutionary models for planetary nebula central stars indicate that, while they still derive energy from nuclear fusion, massive central stars evolve much more rapidly than lower mass central stars. This effect will also tend to decrease the mean abundance observed for bright planetary nebulae by reducing the time during which the planetary nebulae produced by young progenitors are bright. Thus, the similar oxygen abundances in the interstellar medium and in bright planetary nebulae in the Magellanic Clouds (e.g., Richer 1993) reflects the balance between these three effects that the models must strive to attain.

Quantitatively, the abundance parameters that the models must match are set by the planetary nebula population in the LMC. For later comparison with models, the mean oxygen abundances and their dispersions in the Magellanic Clouds, both for the interstellar medium and for planetary nebulae within 2 mag of the PNLF peak, are given in Table 2. Given the number of planetary nebulae within 1 mag of the PNLF peak in the LMC, the models are constrained to predict abundances for the planetary nebulae within 1 mag of the PNLF peak that are within $0.02 \pm 0.10$ dex of the interstellar medium value (99\% confidence limit). For the SMC, the equivalent constraint is $-0.06 \pm 0.22$ dex.

Table 2. Oxygen abundances in the Magellanic Clouds

\begin{tabular}{lcccc}
\hline \hline Galaxy & $\begin{array}{c}\text { Cut-Off } \\
\text { mag) }\end{array}$ & $\begin{array}{c}\text { Mean Abundance } \\
\text { (dex) }\end{array}$ & $\begin{array}{c}\text { Dispersion } \\
\text { (dex) }\end{array}$ & $\begin{array}{c}\text { Number } \\
\text { (in bin) }\end{array}$ \\
\hline LMC & 1 & 8.33 & 0.13 & 20 \\
& 2 & 8.24 & 0.25 & 28 \\
& ISM $^{\mathrm{b}}$ & 8.35 & 0.06 & \\
SMC & & & & 8 \\
& 1 & 8.09 & 0.16 & 8 \\
& 2 & 8.21 & 0.13 & \\
\hline
\end{tabular}

a threshold below the PNLF peak luminosity, all statistics are computed within luminosity bins 1 mag wide whose lower limit is given by the cut-off; values for $m_{5007}^{*}$ are adopted from Jacoby et al. (1990).

b from Russell \& Dopita (1992).

\section{Models of planetary nebula populations in galaxies}

We modelled the planetary nebula populations in galaxies in two steps. The first step was to model the evolution of the host galaxy. This was done using the galaxy modelling code described in Arimoto et al. (1992). This code tracks the consumption of gas, the interstellar medium metallicity, and the numbers of stars formed as a function of time. The second step was to use these data to compute the resulting planetary nebula population, which was done using a code developed specifically for this purpose.

\subsection{Model galaxies}

The galaxy modelling code is well known and has been amply described in the literature (see Arimoto \& Yoshii 1986 for the fundamentals; Arimoto et al. 1992 for subsequent changes). This code divides a galaxy into two parts, a region where star formation occurs and, if gas inflow is allowed, a separate gas reservoir in which no star formation occurs. The basic premise of the code is that, as stars form, they deplete the pool of material available for subsequent star formation. Every stellar generation locks matter into long-lived stars, but each also enriches the interstellar medium in heavy elements by returning processed material from supernovae. This code only implements enrichment from classical type II supernovae (i.e., core collapse in massive stars). As a result, its treatment of oxygen is correct, at least at low metallicities (cf. Maeder 1992), but that of iron is only approximate due to the lack of other supernova sources. A basic assumption is 
that the interstellar medium is always well mixed, so that abundances are uniform throughout and each stellar generation is formed with zero abundance dispersion. At each time step in the evolution of a model galaxy, typically 5 million years, the gas mass fraction, the metallicity of forming stars, and the mass of gas transformed into stars are recorded.

The galaxy models were intended to be very schematic. The galaxies were initially entirely composed of gas with zero metallicity and no gas infall was allowed. Unless specifically noted below, all models use a Salpeter (1955) initial mass function, i.e., $\phi(m)=\mathrm{km}^{-2.35}$, where $m$ is the stellar mass and $k$ is a normalization constant defined such that the integral of the initial mass function is unity. For this project, the only significant modification that was made to the galaxy modelling code was to implement tracking of the interstellar medium oxygen abundance.

\subsection{Model planetary nebula populations}

A separate code, called the planetary nebula population code (PNP code), was developed to compute the global properties of a galaxy's planetary nebula population. The planetary nebula population comprises the planetary nebulae produced by each stellar generation that is old enough to produce planetary nebulae, i.e., age $\gtrsim 10^{8}$ yrs. At each time step, the galaxy modelling code records the mass of gas turned into stars, the metallicity of these stars, and the oxygen abundance in the interstellar medium. The PNP code uses these data for each generation of stars to predict the luminosity function and the mean oxygen abundance for the planetary nebula population. Since these global properties are calculated from the abundances and luminosity functions for planetary nebulae in each stellar generation, the discussion that follows focuses upon this process within a single stellar generation.

\subsubsection{Planetary nebula central stars}

The PNP code uses stellar lifetimes based upon the stellar evolution models of Bressan et al. (1993) and Fagotto et al. (1994a,b). These models are attractive because they span wide mass and metallicity ranges, they are based upon recent opacity calculations, and they incorporate homogeneous input physics. In addition, these models are evolved to the early AGB phase, so there is negligible error in using their lifetimes to that point as their total lifetimes. These stellar lifetimes differ from those used by the galaxy modelling code (Arimoto \& Yoshii 1986), but these more realistic lifetimes are necessary to correctly predict the rate at which each stellar generation produces planetary nebulae (e.g., Renzini \& Buzzoni 1986). For these stellar models, the dividing mass between those stars that go on to become supernovae and those that become white dwarfs is between $5 M_{\odot}$ and $6 M_{\odot}$. Here, all stars with masses below $5.5 M_{\odot}$ are assumed to become planetary nebulae.

For any single stellar generation, its age and metallicity fix the initial masses of its planetary nebula progenitors. At any particular epoch, the mass range for the planetary nebula progenitors in any stellar generation is fixed by the nebular expansion rate, since this determines how long the planetary nebulae remain visible. Planetary nebulae are assumed to be visible so long as their densities exceed $10 \mathrm{~cm}^{-3}$.

Now, knowing the initial masses of the planetary nebula progenitors, the PNP code integrates the initial mass function over the appropriate mass range to determine the mass fraction of this stellar generation that is producing planetary nebulae. The total number of planetary nebulae from each stellar generation is then

number of $\mathrm{PNe}=$

$\underline{\text { mass of stars formed } \times \text { mass fraction of } \mathrm{PN} \text { progenitors }}$. mean mass of $\mathrm{PN}$ progenitors

Given the initial masses of the planetary nebula progenitors in each generation, we assign a planetary nebula central star mass, $M_{\mathrm{c}}$, according to an initial-to-final mass relation

$M_{\mathrm{c}}=a M_{\mathrm{in}}+b M_{\odot}$

where $M_{\text {in }}$ is the initial mass of the planetary nebula progenitor. We determined $a$ and $b$ by comparing the PNLFs from the models with those observed in real galaxies.

The PNP code models the evolution of planetary nebula central stars by interpolating the evolutionary tracks for the hydrogen-burning models of Vassiliadis \& Wood (1994). Fortunately, it is not necessary to interpolate the Vassiliadis \& Wood (1994) models directly, as their temperature and luminosity evolution are completely characterized by the core mass, $M_{\mathrm{c}}$, and the time, $t\left(T_{\max }\right)$, to evolve from a temperature of $10^{4} \mathrm{~K}$ to the maximum temperature. Consequently, it is possible to define scaling parameters, which are functions of $M_{\mathrm{c}}$ and $t\left(T_{\max }\right)$, that transform the evolutionary track for central stars of any mass into a "normalized" evolutionary track. Using the normalized temperature and luminosity evolution as a function of time, in units of $t\left(T_{\max }\right)$, the temporal evolution of a central star of any mass may be calculated by re- scaling the normalized temperature and luminosity according to the value of $M_{\mathrm{c}}$. The evolutionary tracks are only followed to a time of 40000 years after the central star reaches a temperature of $10^{4} \mathrm{~K}$. None of the planetary nebulae in our models is visible for times longer than this.

Figure 3 shows several of the Vassiliadis \& Wood (1994) models along with the corresponding tracks calculated according to the scheme outlined above. Note 
that the interpolated tracks are calculated for the same time points as the Vassiliadis \& Wood (1994) models. The tracks in Fig. 3 are labelled according to the mass of the central star. As this figure demonstrates, the predicted time variation of the luminosity and temperature are in good accord with the models of Vassiliadis \& Wood (1994).

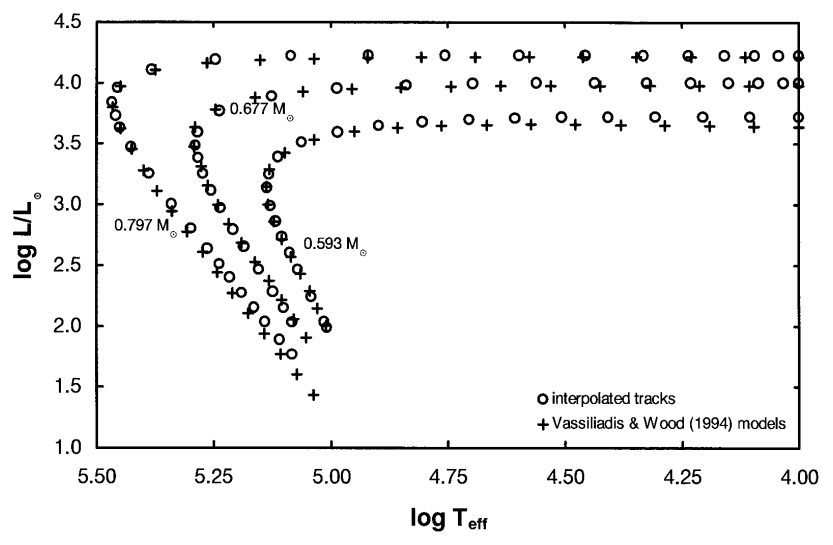

Fig. 3. A comparison of the Vassiliadis \& Wood (1994) models with those computed by the PNP code. The PNP code points are calculated for the same times as the Vassiliadis \& Wood (1994) models. Central star evolution is followed for 40000 years from the time the temperature attains $10000 \mathrm{~K}$

\subsubsection{Nebular $[\mathrm{OIII}] \lambda 5007$ luminosities}

Once the luminosity and temperature of its central star are known, it is relatively straightforward to calculate the $\mathrm{H} \beta$ luminosity of a planetary nebula's shell. The energy distribution for the central star is approximated by a blackbody flux distribution (see Gabler et al. 1991 for justification). Given the central star temperature, $T$, the data in Allen (1973) were interpolated to calculate the fraction, $\theta$, of photons emitted by the star that are capable of ionizing hydrogen:

$$
\begin{aligned}
\log \left(\log \theta^{-1}\right)= & 0.0438 t_{*}^{3}-0.8436 t_{*}^{2} \\
& +3.4366 t_{*}-2.3822 \mathrm{dex},
\end{aligned}
$$

where $t_{*}=\log T$. For a blackbody, the total number of photons emitted can be written as

$N_{\text {phot }}=\frac{1.5210^{11} L}{\sigma T}$ photons s $^{-1}$

(Allen 1973) where $L$ is the stellar luminosity and $\sigma$ is the Stefan-Boltzman constant. In ionization equilibrium, the rate of recombinations must equal the rate of emission of ionizing photons, so the $\mathrm{H} \beta$ luminosity can be determined via (e.g., Osterbrock 1989)

$L(\mathrm{H} \beta)=\frac{\alpha_{\mathrm{H} \beta}^{\mathrm{eff}}\left(\mathrm{H}^{0}, T_{\mathrm{e}}\right)}{\alpha_{\mathrm{B}}\left(H^{0}, T_{\mathrm{e}}\right)} h \nu_{\mathrm{H} \beta} \theta N_{\text {phot }}$ erg s${ }^{-1}$, where $\alpha_{\mathrm{H} \beta}^{\mathrm{eff}}\left(H^{0}, T_{\mathrm{e}}\right)$ and $\alpha_{\mathrm{B}}\left(H^{0}, T_{\mathrm{e}}\right)$ are, respectively, the effective recombination coefficient for the $\mathrm{H} \beta$ line and the hydrogen recombination coefficient summed over all levels except the ground level (Case B). $T_{\mathrm{e}}$ is the nebular electron temperature. We adopted the values of $\alpha_{\mathrm{H} \beta}^{\mathrm{eff}}\left(H^{0}, T_{\mathrm{e}}\right)$ and $\alpha_{\mathrm{B}}\left(H^{0}, T_{\mathrm{e}}\right)$ for a temperature of $10^{4} \mathrm{~K}$ from Osterbrock (1989).

The nebular mass and the optical depth to ionizing photons modify the $\mathrm{H} \beta$ luminosity, however. The nebular geometry is assumed to be a filled spherical shell. The mass of this nebular shell, $M_{\text {neb }}$, is either a fixed maximum of $0.6 M_{\odot}$ or the difference between the progenitor's initial mass and the mass of the central star it produces, whichever is smaller. Whether this nebular mass is sufficient to absorb all of the ionizing photons from the central star depends upon the nebular expansion velocity, $V$, for this fixes the nebular density, $N_{\mathrm{e}}$. At any time, $t$, the nebular density is given by

$N_{\mathrm{e}}=N_{\mathrm{i}}\left(\frac{r_{\mathrm{i}}}{r_{\mathrm{i}}+V t}\right)^{3}$

where $r_{\mathrm{i}}=10^{14} \mathrm{~cm}$ and $N_{\mathrm{i}}=4.1210^{13} \mathrm{~cm}^{-3}$ are the initial radius and density of the nebulae. The initial radius was chosen arbitrarily, while the initial density was fixed by considering the typical values of the $\mathrm{H} \beta$ luminosity, electron density, and expansion velocity of bright planetary nebulae in the Magellanic Clouds. Based upon the complete sample, $\log L(\mathrm{H} \beta)=35.0 \mathrm{dex}, \log N_{\mathrm{e}} \approx 3.67 \mathrm{dex}$ and $V \approx 30 \mathrm{~km} \mathrm{~s}^{-1}$ are typical for the brightest planetary nebulae in the Magellanic Clouds. For a uniform density nebula,

$L(\mathrm{H} \beta)=h \nu_{\mathrm{H} \beta} N_{\mathrm{e}}^{2} \alpha_{\mathrm{H} \beta}^{\mathrm{eff}}\left(H^{0}, T_{\mathrm{e}}\right) \times$ volume,

which yields a typical nebular volume of $3.6910^{52} \mathrm{~cm}^{3}$, or a typical radius of $2.0710^{17} \mathrm{~cm}(0.07 \mathrm{pc})$ for a spherical nebula. For $V=30 \mathrm{~km} \mathrm{~s}^{-1}$, this radius is achieved after $6.910^{10}$ s. Given this time, solving Eq. (7) for $N_{\mathrm{i}}$ yields the initial density given above. The mass that the central star may ionize, $M_{\text {ion }}$, is given by re-arranging Eq. (8), noting the relationship between mass, volume, and density

$M_{\mathrm{ion}}=\frac{L(\mathrm{H} \beta)}{h \nu_{\mathrm{H} \beta} N_{\mathrm{e}} \alpha_{\mathrm{H} \beta}^{\mathrm{eff}}\left(H^{0}, T_{\mathrm{e}}\right)}$.

When the density is sufficiently low that $M_{\text {ion }}>M_{\text {neb }}$, the nebula only absorbs the fraction $M_{\text {neb }} / M_{\text {ion }}$ of the central star's ionizing flux.

Once the $\mathrm{H} \beta$ luminosity is known, assigning an $[\mathrm{OIII}] \lambda 5007 / \mathrm{H} \beta$ ratio fixes the $[\mathrm{OIII}] \lambda 5007$ luminosity. For a planetary nebula whose central star's temperature exceeds $610^{4} \mathrm{~K}$, [OIII $] \lambda 5007 / \mathrm{H} \beta$ is set to the maximum value appropriate for the nebular oxygen abundance, $\{[\mathrm{OIII}] \lambda 5007 / \mathrm{H} \beta\}_{\mathrm{RM} 55}$, given by Eq. (3) of RM95. For central stars with temperatures between $3.410^{4} \mathrm{~K}$ and 
$610^{4} \mathrm{~K}$, the value of $[\mathrm{OIII}] \lambda 5007 / \mathrm{H} \beta$ given by the prescription for hotter stars is reduced by a factor of

$$
\frac{[\mathrm{OIII}] \lambda 5007 / \mathrm{H} \beta}{\{[\mathrm{OIII}] \lambda 5007 / \mathrm{H} \beta\}_{\mathrm{RM} 95}}=\frac{3.610^{-4} T-11.94}{9.66}
$$

to be consistent with the observed correlation between central star temperature and $[\mathrm{OIII}] \lambda 5007 / \mathrm{H} \beta$ (Kaler \& Jacoby 1991; Méndez et al. 1993). This reduction factor accounts for the lower $[\mathrm{OIII}] \lambda 5007 / \mathrm{H} \beta$ ratios that occur while the central stars are evolving from low to high temperatures. The $[\mathrm{OIII}] \lambda 5007 / \mathrm{H} \beta$ ratios for planetary nebulae whose central stars are cooler than $3.410^{4} \mathrm{~K}$ are set to zero.

Given the high initial density, there are occasions when emission of $[\mathrm{OIII}] \lambda 5007$ is quenched by collisional deexcitation. We have implemented this correction according to

quenching factor $=\left(1+\frac{N_{\mathrm{e}} q_{5007}}{A_{5007}}\right)^{-1}$

(Osterbrock 1989) where $q_{5007}$ is the rate of collisional deexcitation per unit volume and $A_{5007}$ is the spontaneous de-excitation coefficient. We adopted the definitions and values for these quantities from Osterbrock (1989).

The PNP code uses two [OIII $] \lambda 5007 / \mathrm{H} \beta$ ratios: a mean value when calculating the luminosity function, and a maximum value when determining the PNLF peak luminosity. When calculating the PNLF peak luminosity, the $[\mathrm{OIII}] \lambda 5007 / \mathrm{H} \beta$ ratio, either $\{[\mathrm{OIII}] \lambda 5007 / \mathrm{H} \beta\}_{\mathrm{RM} 95}$ or the modified value from Eq. (10), is used directly. When calculating the luminosity function, the mean $[\mathrm{OIII}] \lambda 5007 / \mathrm{H} \beta$ ratio for that oxygen abundance is more appropriate, so the maximum ratio, either $\{[\mathrm{OIII}] \lambda 5007 / \mathrm{H} \beta\}_{\mathrm{RM} 95}$ or the modified value from Eq. (10), is reduced by $0.145 \mathrm{dex}$ (see RM95 for details).

\subsubsection{PNLFs and oxygen abundances}

The PNP code follows the temporal evolution of planetary nebulae from each stellar generation through the $\mathrm{H}-\mathrm{R}$ diagram and, using the maximum $[\mathrm{OIII}] \lambda 5007 / \mathrm{H} \beta$ ratio, notes the maximum $[\mathrm{OIII}] \lambda 5007$ luminosity from each generation. The largest sets the maximum $[\mathrm{OIII}] \lambda 5007$ luminosity for the entire population.

Next, using the average $[\mathrm{OIII}] \lambda 5007 / \mathrm{H} \beta$ ratio, the PNP code evolves the planetary nebulae from each stellar generation through the H-R diagram and determines the time intervals during which their luminosities are within $1,2,3,4$, and $5 \mathrm{mag}$ of the maximum [OIII] $\lambda 5007 \mathrm{lu}-$ minosity (the luminosity thresholds). Dividing these five time intervals by the planetary nebula lifetime yields the fraction of their lifetime that the planetary nebulae spend above each luminosity threshold. Since planetary nebulae from a single generation will occupy all possible stages of evolution at any time, the fraction of planetary nebulae with luminosities above any particular threshold is equal to the fraction of their lifetime spent above that threshold. Multiplying the total number of planetary nebulae in this generation by the fraction above each luminosity threshold then gives the actual number of planetary nebulae above each threshold for that generation.

To obtain the luminosity function for the entire planetary nebula population, the process just described is repeated for all stellar generations. The total number of planetary nebulae above each luminosity threshold is then just the sum of the number from each stellar generation. Compared to Monte Carlo-type algorithms, this method is very efficient for computing the luminosity function for a planetary nebula population. However, it is a "high signalto-noise" method in the sense that it does not allow for the fluctuations that occur when small numbers of planetary nebulae are involved.

Once the number of planetary nebulae above each luminosity threshold from each stellar generation are known, the mean abundance and its dispersion within each luminosity bin are calculated.

Throughout this description of the PNP code, we have omitted a complication that occurs in real galaxies: stars of a given age in real galaxies do not all have the same metallicity (e.g., Boesgaard 1989; Rana 1991). The PNP code was designed to allow for an arbitrary metallicity dispersion among the stars formed within a single stellar generation, and simulates the effect of a Gaussian abundance dispersion by splitting the planetary nebula population from each stellar generation into three sub-generations. All planetary nebulae within $1 \sigma$ of the mean, $68 \%$ of all objects, are assigned the abundance from the galaxy code. The remaining planetary nebulae are assigned abundances $1.525 \sigma$ above and below the mean, which is the weighted mean deviation for points beyond $1 \sigma$. The high and low abundance subsets each account for $16 \%$ of the planetary nebulae from each stellar generation. An intrinsic abundance dispersion of 0.13dex within each stellar generation was chosen. This is of the same order of magnitude as the observed abundance dispersion among bright planetary nebulae in the Magellanic Clouds (Table 2; see also Sect. 4).

\subsection{Model results}

Two sequences of model galaxies were calculated. The models in the first sequence, the star-forming sequence, were designed to schematically represent the LMC, the SMC, and the disk of the Milky Way. The four models in this sequence form stars at a constant rate. Their star formation rates were chosen such that their final interstellar medium oxygen abundances spanned the current abundances observed in the Magellanic Clouds and the disk of the Milky Way. The second sequence, the elliptical sequence, emulates the conditions in ellipticals. Most of these models turn various fractions of their initial mass 
into stars during an initial burst of star formation, and then evolve passively, i.e., without any further star formation. The remaining elliptical models undergo a second burst of star formation $10 \mathrm{Gyr}$ after the beginning of the first. All models were evolved to an age of 15 Gyr. (Age is the time elapsed since the beginning of star formation). All of the model galaxies have initial gas masses of $10^{12} M_{\odot}$ except the LMC and SMC models, which have initial gas masses of $610^{9} M_{\odot}$ and $210^{9} M_{\odot}$ respectively (Lequeux 1984; Russell \& Dopita 1992).

The parameters of the LMC model and one of the elliptical models calculated according to the prescription outlined thus far are tabulated in Table 3. (The elliptical model is model R2 from Table 4. Further details will be given below). For comparison, the observed parameters for the LMC and the Henize \& Westerlund (1963) distribution (as modified by Ciardullo et al. 1989b), are also tabulated. Note that the first $2 \mathrm{mag}$ of the LMC PNLF in Table 3 was defined using the LMC's global PNLF, whereas the Jacoby PNLF was used to define the remainder of the LMC PNLF. The interstellar medium oxygen abundance quoted for the elliptical models is the last epoch oxygen abundance, i.e., the oxygen abundance when star formation ceased. For the LMC models, the oxygen abundance is the abundance attained in the interstellar medium at 15 Gyr. For the planetary nebulae, we tabulate the mean oxygen abundances for objects within $1 \mathrm{mag}$ of the PNLF peak and for those with luminosities between 1 and $2 \mathrm{mag}$ below the peak. We normalized the cumulative PNLFs at $3 \mathrm{mag}$ from the PNLF peak, instead of $5 \mathrm{mag}$, since this reduces our sensitivity to the uncertainties in the model parameters. If our prescription of planetary nebula evolution is incorrect, the predicted cumulative number will be systematically worse the lower the luminosity threshold.

In Cols. 4 through 9, Table 3 presents three models of the planetary nebula populations predicted for the LMC and an elliptical galaxy. The three models of the planetary nebula population for each galaxy differ only in the expansion velocity chosen for the planetary nebulae. In the LMC models, almost all planetary nebulae are optically thin if the expansion velocity is $30 \mathrm{~km} \mathrm{~s}^{-1}$, but almost all of them are optically thick if the expansion velocity is $2 \mathrm{~km} \mathrm{~s}^{-1}$. In the elliptical models, all the planetary nebulae are optically thin for expansion velocities of 30 and $5 \mathrm{~km} \mathrm{~s}^{-1}$, but they are all optically thick if the expansion velocity is $2 \mathrm{~km} \mathrm{~s}^{-1}$.

These models demonstrate several successes and drawbacks of the simple model of planetary nebulae described so far. First, in the elliptical models whose planetary nebulae are optically thin (Cols. 7 and 8), the shape of the PNLF is in excellent agreement with the Henize \& Westerlund (1963) luminosity function. Ciardullo (1995) has shown that this luminosity distribution arises naturally from the evolution of optically thin planetary nebulae. Second, for the LMC model whose planetary nebulae expand at $2 \mathrm{~km} \mathrm{~s}^{-1}$ (Col. 4), the shape of the first
4 mag of the PNLF is in respectable agreement with the luminosity function observed in the LMC. This suggests that the shape of the PNLF in the LMC differs from that of the Henize \& Westerlund (1963) luminosity function because the planetary nebulae in the LMC are not optically thin throughout their entire evolution. Finally, the planetary nebulae in the elliptical model are able to achieve the PNLF peak luminosity observed in the LMC, if the planetary nebulae are optically thick to the central star's ionizing radiation (Col. 9). Thus, to achieve the observed PNLF peak luminosity in ellipticals, there is no need to invoke a subset of planetary nebulae containing more massive central stars (Sect. 2.3). This is not surprising, for attaining the PNLF peak luminosity requires only a modest central star mass. As has been found previously (e.g., Dopita et al. 1992), our model planetary nebulae attain their maximum [OIII $\lambda(5007$ luminosity when the central star's temperature is approximately $70000 \mathrm{~K}$. By Eq. (4), the fraction of ionizing radiation at this temperature is 0.56 . Given $\log L(5007)=36.20 \mathrm{dex}$ and $\log I(5007) / I(\mathrm{H} \beta)=1.14$ dex for the brightest planetary nebulae in the Magellanic Clouds (RM95), and Eqs. (5) and (6), the necessary stellar luminosity is $1.1210^{37} \mathrm{erg} \mathrm{s}^{-1}$ or $2925 L_{\odot}$. According to Eq. (5) of Vassiliadis \& Wood (1994), this corresponds to the luminosity of a central star with a mass of approximately $0.55 M_{\odot}$. Thus, while the model of planetary nebula evolution described so far is able to reproduce many of the observed properties of planetary nebula populations, these properties are not simultaneously achieved using a single set of model parameters.

There are two significant difficulties with the models in Cols. 4 through 9, and these indicate the directions in which solutions should be sought. First, the PNLF peak luminosity varies: it is too bright in the LMC models and too faint in the elliptical galaxy models. As noted above, the peak luminosity in the elliptical models can be brought into agreement with that observed if the nebular shells absorb all of the central star's ionizing radiation. Hence, their densities cannot be too low. Second, although this is not apparent from Table 3 , the brightest planetary nebulae in the LMC model are much denser and have lower ionized masses than their observed counterparts. Thus, the massive central stars in the LMC models evolve too quickly compared to the nebular envelopes, whereas the low mass central stars in the elliptical models evolve too slowly compared to the nebular envelopes. This evolution time scale difficulty may be addressed in equivalent ways through either an expansion velocity law that depends upon the central star mass or an adjustment of the central star evolution time scales. However, neither of these solutions will eliminate the already overluminous planetary nebulae in the LMC models, unless the expansion velocities or evolution time scales are large enough to make the planetary nebulae optically thin. 
Table 3. Parameters of the planetary nebula population: Naïve models

\begin{tabular}{|c|c|c|c|c|c|c|c|c|c|c|c|c|c|c|c|c|}
\hline \multirow[b]{2}{*}{ (1) } & \multirow{2}{*}{$\begin{array}{l}\text { LMC } \\
(2)\end{array}$} & \multirow{2}{*}{$\begin{array}{c}\text { H\&W } \\
(3)\end{array}$} & \multicolumn{3}{|c|}{ LMC models } & \multicolumn{3}{|c|}{ R2 elliptical models } & \multirow{2}{*}{$\begin{array}{l}\text { LMC } \\
(10)\end{array}$} & \multirow{2}{*}{$\begin{array}{c}\mathbf{R} 2 \\
(11)\end{array}$} & \multirow{2}{*}{$\begin{array}{c}\text { LMC } \\
(12)\end{array}$} & \multirow{2}{*}{$\begin{array}{c}\mathrm{R} 2 \\
(13)\end{array}$} & \multirow{2}{*}{$\begin{array}{c}\text { LMC } \\
(14)\end{array}$} & \multirow{2}{*}{$\begin{array}{c}\mathrm{R} 2 \\
(15)\end{array}$} & \multirow{2}{*}{$\begin{array}{c}\text { LMC } \\
(16)\end{array}$} & \multirow{2}{*}{$\begin{array}{c}\mathrm{R} 2 \\
\text { (17) }\end{array}$} \\
\hline & & & (4) & (5) & $(6)$ & (7) & $(8)$ & (9) & & & & & & & & \\
\hline $\bar{M}_{\mathrm{vel}}\left(\mathrm{M}_{\odot}\right)$ & & & & & & & & & & & & & 1.0 & 1.0 & 1.0 & 1.0 \\
\hline$M_{\text {cov }}\left(\mathrm{M}_{\odot}\right)$ & & & & & & & & & 0.548 & 0.548 & 0.548 & 0.548 & 0.548 & 0.548 & 0.548 & 0.548 \\
\hline$V\left(\mathrm{~km} \mathrm{~s}^{-1}\right)$ & & 30 & 5 & 2 & 30 & 5 & 2 & 30 & 30 & Eq. 13 & Eq. 13 & Eq. 13 & Eq. 13 & Eq. 14 & Eq. 14 & \\
\hline $\log L(5007)_{\max } 36.20$ & & 36.80 & 36.53 & 36.37 & 33.13 & 35.47 & 36.22 & 36.15 & 33.13 & 36.17 & 36.21 & 36.17 & 36.16 & 36.17 & 36.21 & \\
\hline $\log N(3 \mathrm{mag})$ & 1.89 & & 0.62 & 2.26 & 3.26 & 4.85 & 5.29 & 5.92 & 0.92 & 4.85 & 3.01 & 5.85 & 2.81 & 5.56 & 1.64 & 3.95 \\
\hline$f(1 \mathrm{mag})$ & $0.30 \pm 0.07$ & 0.18 & 0.22 & 0.20 & 0.24 & 0.12 & 0.15 & 0.33 & 0.22 & 0.12 & 0.13 & 0.19 & 0.18 & 0.14 & 0.28 & 0.17 \\
\hline$f(2 \mathrm{mag})$ & $0.64 \pm 0.12$ & 0.53 & 0.56 & 0.57 & 0.67 & 0.50 & 0.51 & 0.69 & 0.56 & 0.50 & 0.53 & 0.61 & 0.58 & 0.54 & 0.68 & 0.56 \\
\hline$f(3 \mathrm{mag})$ & $1.00 \pm 0.16$ & 1.00 & 1.00 & 1.00 & 1.00 & 1.00 & 1.00 & 1.00 & 1.00 & 1.00 & 1.00 & 1.00 & 1.00 & 1.00 & 1.00 & 1.00 \\
\hline$f(4 \mathrm{mag})$ & $1.36 \pm 0.20$ & 1.65 & 1.64 & 1.56 & 1.28 & 1.71 & 1.69 & 1.34 & 1.63 & 1.71 & 1.37 & 1.37 & 1.43 & 1.63 & 1.30 & 1.59 \\
\hline$f(5 \mathrm{mag})$ & $1.79 \pm 0.25$ & 2.52 & 4.28 & 4.61 & 5.75 & 2.65 & 2.60 & 3.62 & 3.95 & 2.65 & 2.91 & 3.07 & 3.19 & 2.96 & 3.27 & 3.34 \\
\hline $12+\log (O / H)_{\mathrm{ISM}}$ & 8.35 & & 8.29 & 8.29 & 8.29 & 9.09 & 9.09 & 9.09 & 8.29 & 9.09 & 8.29 & 9.09 & 8.29 & 9.09 & 8.29 & 9.09 \\
\hline $12+\log (O / H)_{1} \operatorname{mag}$ & 8.33 & & 8.27 & 8.31 & 8.31 & 8.92 & 9.00 & 8.91 & 8.26 & 8.92 & 8.24 & 8.90 & 8.25 & 9.02 & 8.21 & 8.90 \\
\hline$\sigma(O / H)_{1 \mathrm{mag}}$ & 0.13 & & 0.07 & 0.14 & 0.12 & 0.12 & 0.15 & 0.23 & 0.07 & 0.12 & 0.12 & 0.22 & 0.12 & 0.16 & 0.09 & 0.14 \\
\hline $12+\log (O / H)_{2} \mathrm{mag}$ & 8.24 & & 8.26 & 8.26 & 8.18 & 8.74 & 8.79 & 8.66 & 8.25 & 8.74 & 8.08 & 8.66 & 8.16 & 8.85 & 8.14 & 8.75 \\
\hline$\sigma(O / H)_{2} \operatorname{mag}$ & 0.25 & & 0.07 & 0.15 & 0.19 & 0.22 & 0.26 & 0.36 & 0.07 & 0.22 & 0.20 & 0.36 & 0.16 & 0.26 & 0.13 & 0.21 \\
\hline
\end{tabular}

Notes to Table 3: $M_{\mathrm{vel}}$ is the initial mass below which the expansion velocity from Eq. (13) is held fixed; $M_{\text {cov }}$ is the central star mass above which the nebular covering factor is less than unity; $V$ is the nebular expansion velocity; $L(5007)_{\max }$ is the PNLF peak luminosity; $N(3 \mathrm{mag}$ ) is the number of planetary nebulae within $3 \mathrm{mag}$ of the PNLF peak; $f(X \mathrm{mag})$ is the fraction of planetary nebulae within $X$ mag of the PNLF peak; $12+\log (O / H)_{\text {ISM }}$ is the oxygen abundance in the interstellar medium: at a time of $15 \mathrm{Gyr}$ for the LMC models and at $1 \mathrm{Gyr}$ for the elliptical models; $12+\log (O / H)_{X}$ mag is the mean oxygen abundance for planetary nebulae with luminosities between $(X-1)$ and $X$ mag of the PNLF peak.

Table 4. Parameters of the planetary nebula population: Final models

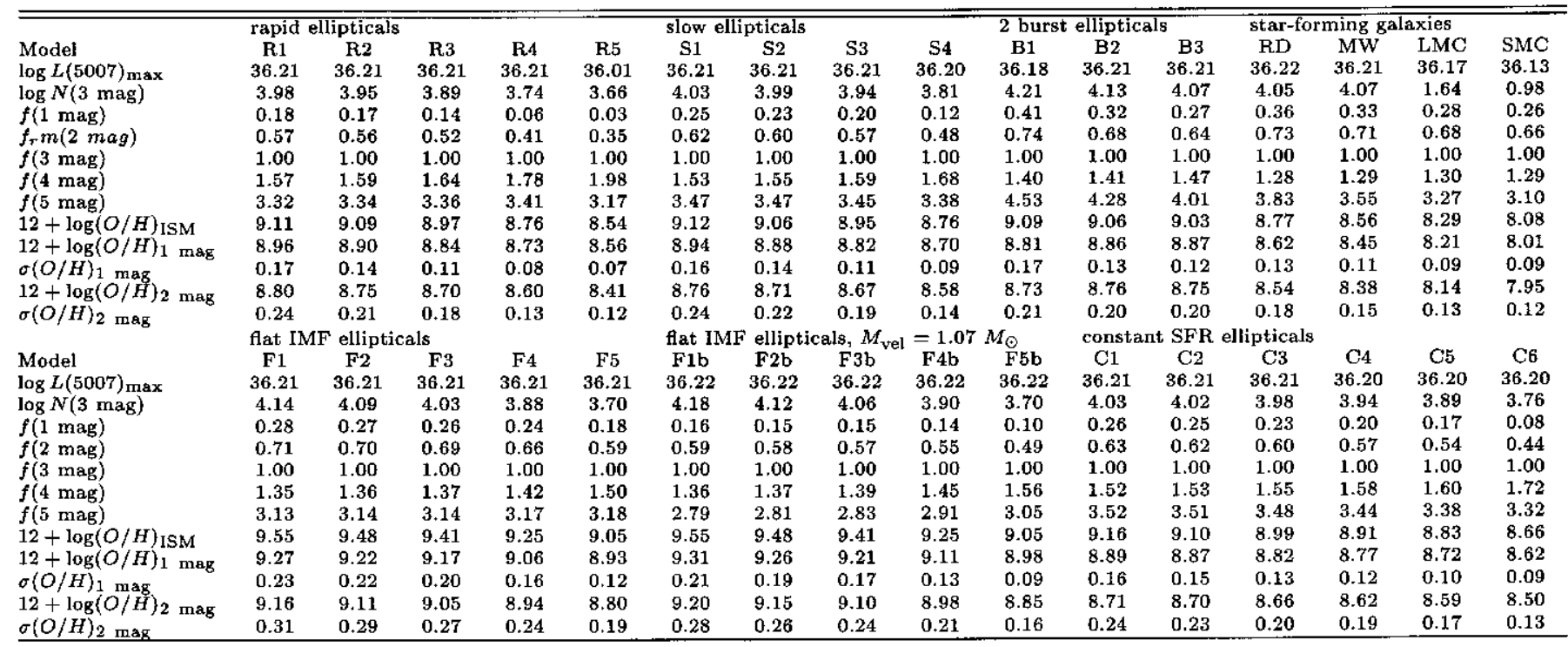

Notes to Table 4: $L(5007)_{\max }$ is the PNLF peak luminosity; $N$ (3 mag) is the number of planetary nebulae within 3 mag of the PNLF peak; $f(X \mathrm{mag})$ is the fraction of planetary nebulae within $X$ mag of the PNLF peak; $12+\log (O / H)_{\text {ISM }}$ is the oxygen abundance in the interstellar medium; $12+\log (O / H)_{X \text { mag }}$ is the mean oxygen abundance for planetary nebulae with luminosities between $(X-1)$ and $X$ mag of the PNLF peak.

There are two obvious possibilities for reducing the luminosities of the planetary nebulae in the LMC models. The nebulae could be optically thin. It is possible to devise a relation between expansion velocity and central star mass such that the maximum [OIII] $\lambda 5007$ luminosity achieved is equal to that observed in the LMC. However, the PNLFs for such LMC models are too steep, being very similar the PNLFs for the elliptical models (i.e., following the Henize \& Westerlund (1963) luminosity distribution). Another solution is to impose a relation between central star mass (or the initial mass) and a nebular covering factor. In this picture, more massive planetary nebula progenitors produce more asymmetric nebulae or nebulae with smaller filling factors. For convenience, we have implemented this covering factor as a function of the central star mass

covering factor $=\frac{M_{\mathrm{cov}}-0.5}{M_{\mathrm{c}}-0.5}$

where $M_{\mathrm{c}}$ is the planetary nebula central star mass and we have set $M_{\text {cov }}=0.548 M_{\odot}$. Note that the nebular mass is not affected by the covering factor, the matter is simply distributed asymmetrically. Columns 10 and 11 of Table 3 present the same LMC and elliptical models as Cols. 4 
and 7 , respectively, but implementing a covering factor as given by Eq. (12). Adding a covering factor only affects the LMC model since almost all of the central stars in the elliptical model have masses below $M_{\text {cov }}$. For the model of the LMC, adding the covering factor reduces the PNLF peak luminosity to roughly that observed.

The brightest planetary nebulae in the LMC model (Col. 10) still have higher densities and lower ionized masses than are observed, while the planetary nebulae in the elliptical model (Col. 11) are still too faint. Both of these problems may be addressed through an expansion velocity law. After much trial and error, we found that the properties of the brightest planetary nebulae in the LMC model matched those observed if their expansion velocities allowed them to begin leaking ionizing photons shortly after attaining their maximum luminosity. (Here, and in the following, leakage of photons refers to those directions in which there is matter. The nebulae always leak ionizing photons over that fraction of a sphere where there is no matter). Given the evolution time scales of the Vassiliadis \& Wood (1994) models, the necessary expansion velocities, $V_{\mathrm{t}}$, at which the nebulae begin leaking photons shortly after attaining maximum luminosity are

$V_{\mathrm{t}}=2750 M_{\mathrm{c}}^{11.65} \mathrm{~km} \mathrm{~s}^{-1}$.

The models shown in Cols. 12 and 13 of Table 3 incorporate planetary nebulae with these expansion velocities. The densities and ionized masses of the brightest planetary nebulae in the LMC model in Col. 12 are similar to those observed. The LMC and elliptical models also now have similar PNLF peak luminosities. The peak luminosities differ only because the mean oxygen abundance is higher in the elliptical model, resulting in a slightly larger $[\mathrm{OIII}] \lambda 5007 / \mathrm{H} \beta$ ratio. In addition, the oxygen abundances for the first two luminosity bins in the model of the LMC now differ, as observed.

However, the PNLF in the elliptical model in Col. 13 is flatter than the Henize \& Westerlund (1963) luminosity function. Its PNLF is too flat because the planetary nebulae are all optically thick until after they attain their maximum luminosity. We can make the PNLF steeper by imposing a lower limit upon $V_{\mathrm{t}}$. If there is a lower limit to $V_{\mathrm{t}}$, then, as the central star masses decrease, the planetary nebulae begin leaking ionizing photons earlier in their evolution. Consequently, planetary nebulae with lower mass central stars attain lower maximum luminosities and fade sooner, reducing the number of bright planetary nebulae and increasing the number of faint ones, both of which make the PNLF steeper. To allow us to vary both $V_{\mathrm{t}}$ and the initial-to-final mass relation independently, we implemented $V_{\mathrm{t}}$ as a cut-off velocity corresponding to an initial mass, $M_{\mathrm{vel}}$. Experiment showed that a cutoff corresponding to an initial mass $M_{\mathrm{vel}}=1 M_{\odot}$ worked well. Models incorporating this lower limit to $V_{\mathrm{t}}$ are found in Cols. 14 and 15 of Table 3 . As expected, the PNLF for the elliptical model is now steeper, and in good agreement with the Henize \& Westerlund (1963) luminosity function. The PNLF peak luminosity in this model has dropped, however, indicating that even the brightest planetary nebulae are now somewhat optically thin to ionizing radiation. The shape of the LMC PNLF is now also closer to that observed, though the fraction of objects in the highest luminosity bin is still too low.

The only important discrepancy remaining between observation and the models presented in Cols. 14 and 15 is the shape of the PNLF in the LMC model. (Note that we are concentrating only on the first 4 mag of the PNLF). Considering the very strong dependence of $V_{\mathrm{t}}$ upon central star mass (Eq. 13), the expansion velocities for the more massive model planetary nebulae are very high, so their lifetimes are short. Indeed, the expansion velocities predicted by Eq. (13) are not realistic expansion velocities. The predicted velocities for the planetary nebulae with massive central stars exceed those observed in the Magellanic Clouds by over a factor of ten. (Typically LMC planetary nebulae span the 20 to $80 \mathrm{~km} \mathrm{~s}^{-1}$ velocity range, e.g., Dopita et al. 1988). The model of the LMC has too few planetary nebulae in the brightest luminosity bin because the planetary nebulae produced by the youngest progenitors evolve too quickly. The simplest remedy is to re-map the expansion velocities to a smaller range. Consequently, we implemented the expansion velocity law, $V_{\exp }$,

$V_{\exp }=66 M_{\mathrm{c}}^{2} \mathrm{~km} \mathrm{~s}^{-1}$.

To use the expansion velocities from Eq. (14), we had to modify the evolution time scales for the central stars by a factor of $V_{\mathrm{t}} / V_{\exp }$ in order to preserve the correct density and ionized masses of the brightest planetary nebulae in the LMC model. Although our choice of Eq. (14) is arbitrary, it represents a compromise. As the dependence upon the central star mass becomes stronger, the bright end of the PNLF in the LMC model becomes steeper, and the mean oxygen abundance for the planetary nebulae in the highest luminosity bin increases. Equation (14) allows a good fit to both the PNLF and the abundances for the brightest planetary nebulae. Because Eq. (14) is much less sensitive to central star mass than Eq. (13), the evolution time scales for massive central stars are increased considerably while those for low mass central stars are shortened. However, central stars of high mass still evolve faster than those of low mass. Models incorporating these new expansion velocities and corrected central star evolution time scales are found in Cols. 16 and 17 of Table 3. These changes have very little effect upon the elliptical model because its central stars span such a narrow mass range, but they modify the shape of the PNLF for the LMC model, bringing it into excellent agreement with the observed PNLF.

The initial-to-final mass relation we adopted for the models in Table $3, M_{\mathrm{c}}=0.094 M_{\mathrm{in}}+0.462 M_{\odot}$, is steeper 
than that tabulated by Weidemann (1987). A fit to his table yields $M_{\mathrm{c}}=0.054 M_{\text {in }}+0.492 M_{\odot}$ for solar metallicity. Our slope was constrained to be significantly steeper than Weidemann's (1987) in order to produce planetary nebulae near the PNLF peak in the most metal-poor elliptical models (see Table 4). Physically, we require a steeper slope because the mass range spanned by our planetary nebula progenitors is narrower than that spanned by the models Weidemann (1987) used to derive his white dwarf masses. Adding a metallicity term to the initial- to-final mass relation has only a very minor effect, both within a single model and differentially between models of different mean metallicity.

The models in Cols. 16 and 17 of Table 3 represent our final prescription for planetary nebula evolution, and it is this prescription we adopt for the models that follow. Although our models predict more faint planetary nebulae than are observed or predicted, they otherwise reproduce the first 4 mag of the PNLFs in galaxies with and without star formation, and also populate the bright end of the PNLF in the LMC model with planetary nebulae having physical properties and abundances like those observed.

The models in Cols. 16 and 17 of Table 3 are complex. To our simple initial prescription, we had to add a nebular covering factor, an expansion velocity law, and adjust the evolution time scales of the central stars. Conceivably, the covering factor could be related to the manner in which matter is lost from the planetary nebula progenitor, which is very poorly constrained by observations. Asymmetry exists in a large fraction of post-AGB stars, for their light is polarized (e.g., Trammell et al. 1994; Johnson \& Jones 1991). Although the total number of objects observed in these studies is small, it would be interesting to learn whether the degree of polarization is correlated with luminosity. The need for an expansion velocity law and an adjustment of the evolution time scale for the central star amount to coordinating the evolution of the central star and its surrounding nebular envelope. Observationally, the precise form of this connection is unknown, but its existence seems likely, e.g., the correlation of expansion velocities with the central star temperature and luminosity observed for Magellanic Cloud planetary nebulae (Dopita \& Meatheringham 1990). Observationally, it is straightforward to estimate the evolution time scale of the nebular shell from expansion velocities, but it is much more difficult to study the evolution time scale of the central star. In their study of the ages of central stars (most of them massive) and their surrounding nebulae, McCarthy et al. (1990) found that many nebular shells appear to be older than their central stars. To reconcile the nebular and stellar ages, they favoured slower central star evolution, arguing that massive central stars would evolve more slowly if they stopped losing mass at a fixed envelope mass instead of at a fixed effective temperature. This would lead to larger envelope masses for massive central stars, which would slow their evolution (e.g., Schönberner 1987). The large fraction of massive central stars among those studied by Méndez et al. (1992) also argues, qualitatively, that the lifetimes of these stars is not as short as models predict. Clearly, the need for a covering factor and the connection between the nebular shell and the central star represent the greatest liabilities of the models presented here. This is not especially surprising, perhaps. Quantitatively, observations provide only very loose constraints upon the way planetary nebula progenitors lose matter, how or when it stops, and whether the subsequent evolution of the central star and nebular envelope are coupled.

The parameters for the remaining galaxy models are presented in Table 4. The format of Table 4 is identical to that of Table 3, though we have suppressed listing $M_{\mathrm{cov}}$, $M_{\mathrm{vel}}$, and the velocity law since these are all identical to those of the final two models in Table 3. In Table 4, there are five series of elliptical galaxy models. The models within a single series differ from one another by the fraction of their initial mass that they convert into stars. The model series differ from one another by their histories of star formation. The first series of elliptical galaxy models is the "rapid" elliptical series (models $\mathrm{R} X$, where $X=\{1,2, \ldots\}$, in Table 4$)$. These model galaxies formed all of their stars in the first billion years of their existence and subsequently evolved passively. The second series, the "slow" ellipticals (SX in Table 4), is identical to the rapid series, but the models in this series formed their stars over a 4 Gyr period, then evolved passively. The star formation rate in the rapid and slow elliptical series decreased exponentially with time, and was chosen so that the distribution of stellar ages was the same in all the models in each series. The models in the third elliptical series, the "constant SFR" ellipticals (CX in Table 4), also formed their stars over a $4 \mathrm{Gyr}$ period, but at a constant star formation rate. The models in the fourth series, the two-burst ellipticals (BX in Table 4), also employed a constant star formation rate, but they formed their stars in two $1 \mathrm{Gyr}$ bursts beginning at ages of 0 and 10 Gyr. Models B1, B2, and B3, formed $50 \%, 33 \%$, and $20 \%$, respectively, of their total stellar mass during the second burst. The final series of elliptical models, the "flat IMF" ellipticals (FX in Table 4), is identical to the rapid series, but it was computed using an initial mass function with a slope of -2.05 instead of the Salpeter (1955) value of -2.35 .

Inspection of the PNLFs for the elliptical models in Table 4 shows that they vary. We chose our model parameters so that the PNLFs for the elliptical models with last epoch oxygen abundances between one and two times the solar value had the same shape as the Henize \& Westerlund (1963) luminosity function (the two-burst models excepted). On either side of this abundance range, however, our PNLFs for the elliptical models deviate systematically from the Henize \& Westerlund (1963) function. Observationally, the shape of the PNLF in environments where the metallicity exceeds that in the bulge of M 31 is unknown. As for the lower metallicity regime, 
based upon the data from Ciardullo et al. (1989b), the first 3 mag of NGC 205's PNLF is in excellent agreement with the Henize \& Westerlund (1963) luminosity function. Some of the variation in the models occurs because of limitations to the ingredients. Principally, the abundancedependence of the stellar lifetimes is held constant for metallicities exceeding solar metallicity, and the variation of the $[\mathrm{OIII}] \lambda 5007 / \mathrm{H} \beta$ ratio is fixed for oxygen abundances exceeding $12+\log (\mathrm{O} / \mathrm{H})=9.01$, where the calibrating data runs out (RM95). We found that we could obtain better agreement for the models of low metallicity ellipticals by adopting a smaller value for $M_{\text {vel }}$ or a larger zero point in our initial-to-final mass relation. In Table 4 we present a second series of flat IMF elliptical models (the FX b series) that was calculated with a larger value of $M_{\mathrm{vel}}$, $M_{\mathrm{vel}}=1.07 M_{\odot}$ and their PNLFs are in good agreement with the Henize \& Westerlund (1963) luminosity function. Our choice of $M_{\mathrm{vel}}=1.0 M_{\odot}$ is a compromise, allowing a reasonable fit over a wide range of metallicity.

Although the PNLF shape in the elliptical models depends somewhat upon the model parameters, the abundances predicted for the brightest planetary nebulae are not too sensitive to these parameters. Considering the two series of flat IMF ellipticals, there is a systematic offset in the abundances of the brightest planetary nebulae by only $\sim 0.05$ dex (see Fig. 5). Similarly, had we chosen a value of $M_{\text {vel }}$ or our initial-to-final mass relation to achieve a good fit to the PNLF for the low metallicity ellipticals, the oxygen abundances for the brightest planetary nebulae in the $\mathrm{R} X, \mathrm{~S} X, \mathrm{C} X$, and $\mathrm{F} X$ models would decrease by $\sim 0.04$ dex.

In Fig. 4, we plot the PNLFs for the R2, S2, C2, F5, and B1 elliptical models, which all have last epoch oxygen abundances between $9.05<12+\log (\mathrm{O} / \mathrm{H})<9.10$ dex. Clearly, our models predict that the shape of the PNLF is insensitive to the history of star formation in an elliptical galaxy if star formation ended long ago. The PNLF for model B1 is flatter at the bright end than the other models shown here, but it is also an extreme case, having formed half of its stars only 5 Gyr before the present. Were the fraction of late-forming stars smaller, as in models B2 and B3, the differences seen in Fig. 4 would be smaller as well.

There are four star-forming galaxy models in Table 4 . There is a slight systematic change in the shape of the PNLF in the star-forming galaxies as the final metallicity increases. The reduced sensitivity of the $[\mathrm{OIII}] \lambda 5007 / \mathrm{H} \beta$ ratio to oxygen abundance at high abundance is responsible for this change in the PNLF shape. Because the $[\mathrm{OIII}] \lambda 5007 / \mathrm{H} \beta$ ratio changes more slowly with increasing oxygen abundance at high abundance, there are smaller luminosity differences between the planetary nebula offspring from stellar generations of different metallicity. Thus, a larger fraction of all stellar generations produce planetary nebulae whose maximum luminosities fall in the

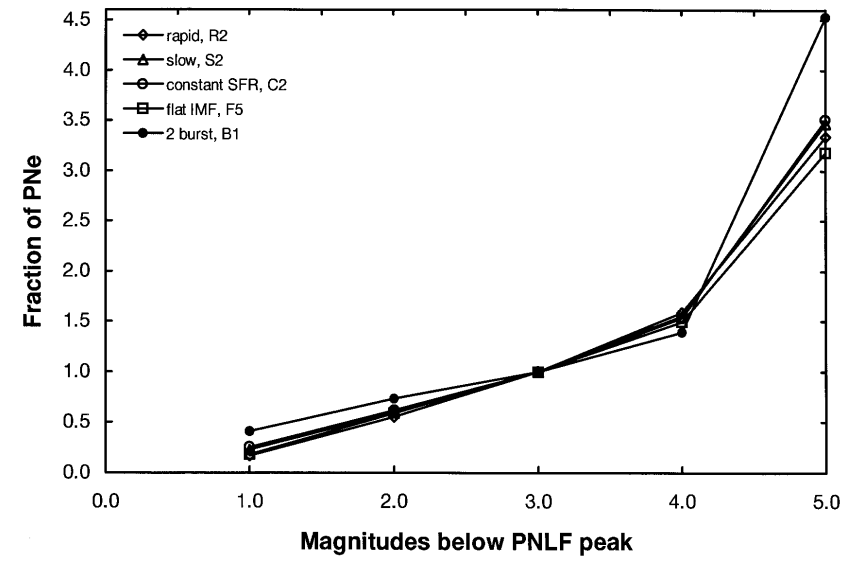

Fig. 4. The PNLFs for the R2, S2, C2, F5, and B1 elliptical galaxy models (Table 4). These models predict that the history of star formation has a negligible effect upon the shape of the PNLF in elliptical galaxies, unless they have suffered a significant, recent episode of star formation

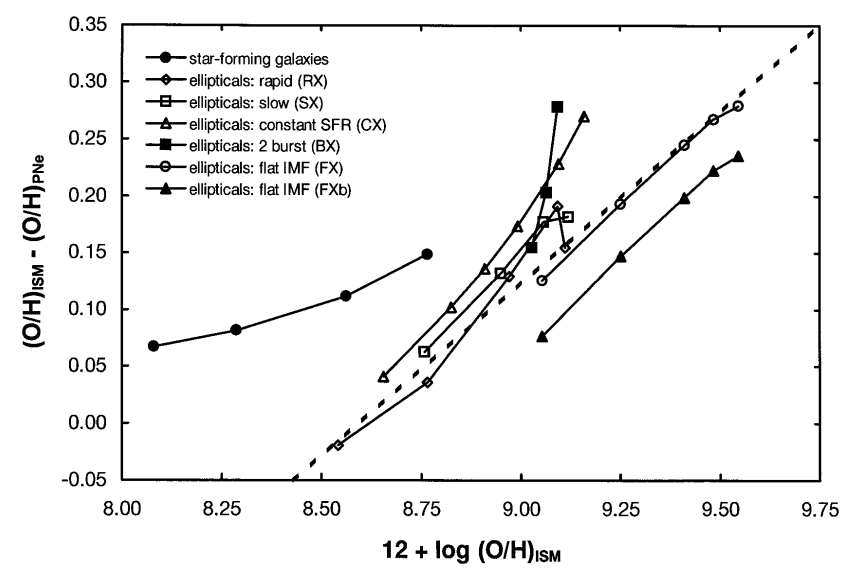

Fig. 5. The abundance gap for all of the models from Table 4. The dashed line is a straight line fit to the rapid $(\mathrm{R} X)$ and flat IMF (FX) model series and is given by Eq. (15). Although the abundance gaps depend primarily upon the final oxygen abundance attained in the interstellar medium, there is also some dependence upon the history of star formation

highest luminosity bin, so the PNLFs become flatter at the bright end.

\subsubsection{Specific densities for the model planetary nebula} populations

These models do not fit the observed trends in planetary nebula specific density (Sect. 2.3). Observations show that the planetary nebula specific density decreases in redder galaxies. Between models of different metallicity, however, the planetary nebula specific density increases in redder galaxies. It is unclear whether this is due to a deficiency in our model of planetary nebula evolution or to 
modes of stellar evolution that are not included in our process of planetary nebula production. In particular, a process that prevents larger fractions of stars from producing planetary nebulae at higher metallicities, e.g., the AGBmanqué route, could help solve several problems. Such a planetary nebula "sink" would not only help reverse the specific density trends found in our models, but it would also help to avoid the metallicity-dependent changes in the PNLF shape discussed earlier.

\section{The abundance gap}

A careful inspection of Table 4 reveals that the difference between the oxygen abundance in the interstellar medium and the mean abundance for bright planetary nebulae (henceforth, the abundance gap) is a function of the interstellar medium abundance. (Here, bright planetary nebulae are those within $1 \mathrm{mag}$ of the PNLF peak). The abundance gap is displayed as a function of the interstellar medium oxygen abundance in Fig. 5. The abundance gap is plotted for ages of 15 Gyr for all the models from Table 4 . The different model series are described in the figure legend. The dashed line in Fig. 5 is a straight line fit to the rapid and flat IMF elliptical series (the $\mathrm{R} X$ and $\mathrm{F} X$ series from Table 4 ), and is given by

$$
\begin{aligned}
\left(x_{\mathrm{ISM}}-x_{\mathrm{PNe}}\right)_{1 \mathrm{mag}}= & (0.302 \pm 0.018) x_{\mathrm{ISM}} \\
& +(-2.60 \pm 0.17)
\end{aligned}
$$

where $x_{\mathrm{ISM}}=12+\log (\mathrm{O} / \mathrm{H})_{\mathrm{ISM}}$ and $x_{\mathrm{PNe}}=12+$ $\log (\mathrm{O} / \mathrm{H})_{\mathrm{PNe}}$.

Two general trends are apparent in Fig. 5. First, within a model series, the abundance gap increases as the oxygen abundance in the interstellar medium increases. Second, the abundance gap increases along the sequence of model series from fast elliptical, slow elliptical, constant SFR elliptical, to star forming galaxies, which is a sequence in which the mean time scale for star formation increases. Thus, the abundance gap also increases as the star formation time scale increases.

The first trend has a simple origin. As the oxygen abundance increases, the abundance sensitivity of the $[\mathrm{OIII}] \lambda 5007 / \mathrm{H} \beta$ ratio decreases. Consequently, there is less luminosity differentiation among the planetary nebulae from different stellar generations, allowing a larger fraction of all stellar generations to contribute to the bright planetary nebula population. As a result, the spread in the oxygen abundances among bright planetary nebulae increases as the oxygen abundance in the interstellar medium increases, causing a departure of the mean oxygen abundance for the bright planetary nebulae from the interstellar medium abundance.

The reason that the abundance gap increases as the time scale for star formation increases is due to the nebular covering factor. At any epoch, if we ignore the effect of metallicity upon stellar lifetimes, planetary nebula central stars with masses of $M_{\text {cov }}$ arise from stellar generations that were born at some specific time, $t_{\text {cov }}$. Thus, the longer star formation continues beyond $t_{\text {cov }}$, the larger is the fraction of planetary nebulae with central stars whose masses exceed $M_{\text {cov }}$ and whose luminosities are effectively identical on account of the covering factor. For planetary nebulae whose central star masses exceed $M_{\text {cov }}$, the dependence of the $[\mathrm{OIII}] \lambda 5007 / \mathrm{H} \beta$ ratio upon oxygen abundance and the expansion velocity are the only properties differentiating their [OIII] $\lambda 5007$ luminosities. The effect of the oxygen abundance is not strong, however. At a given optical depth, all planetary nebulae with oxygen abundances $12+\log (\mathrm{O} / \mathrm{H}) \geq 7.41$ dex will attain maximum [OIII $] \lambda 5007$ luminosities within $1 \mathrm{mag}$ of the PNLF peak (see Eq. (3) of RM95), if they contain a central star with a mass of at least $M_{\text {cov }}$. Consequently, the longer star formation persists, the wider the abundance range for the brightest planetary nebulae becomes, and the more the mean abundance departs from that in the interstellar medium. This effect is muted in the elliptical models because of the cutoff in the expansion velocities. Except for the two-burst ellipticals, the planetary nebula progenitors in these models all have masses below $M_{\mathrm{vel}}$, so the youngest, most metal-rich progenitors are also the most optically thick, which biases their contribution to the population of bright planetary nebulae.

The abundance gap is not sensitive to the yield of oxygen from dying stars. The value for the yield of oxygen affects how far chemical evolution must proceed if a given interstellar medium abundance is to be reached. With a higher value for the yield, chemical evolution must stop at a larger gas fraction if the interstellar medium is to have the same final oxygen abundance. The yield is controlled by the slope of the initial mass function, which fixes the fraction of stars that return newly synthesized oxygen to the interstellar medium. In Fig. 5, the $\mathrm{R} X$ and $\mathrm{F} X$ elliptical model series have similar abundance gaps in the abundance range where they overlap, even though their final gas fractions are very different.

The magnitude of the abundance gap is almost independent of the intrinsic abundance dispersion adopted for each stellar generation (Sect. 3.2.3). Varying the intrinsic dispersion from 0.066dex to 0.20dex decreases the abundance gap by at most 0.03 dex. Thus, the abundance gap is not an artifact of our particular choice for the intrinsic abundance dispersion among stars from each stellar generation.

Figure 6 shows the temporal behaviour of the abundance gaps in the star- forming models. The solid line is a fit to the abundance gaps for the 15 Gyr points, and is given by

$x_{\mathrm{ISM}}-x_{\mathrm{PNe}}=(0.118 \pm 0.016) x_{\mathrm{ISM}}+(-0.89 \pm 0.13)(16)$

where $x_{\mathrm{ISM}}$ and $x_{\mathrm{PNe}}$ have the same meanings as in Eq. (15). For comparison, the fit to the $15 \mathrm{Gyr}$ points for the $\mathrm{R} X$ and $\mathrm{F} X$ elliptical models (Eq. 15) is also shown in Fig. 6. 


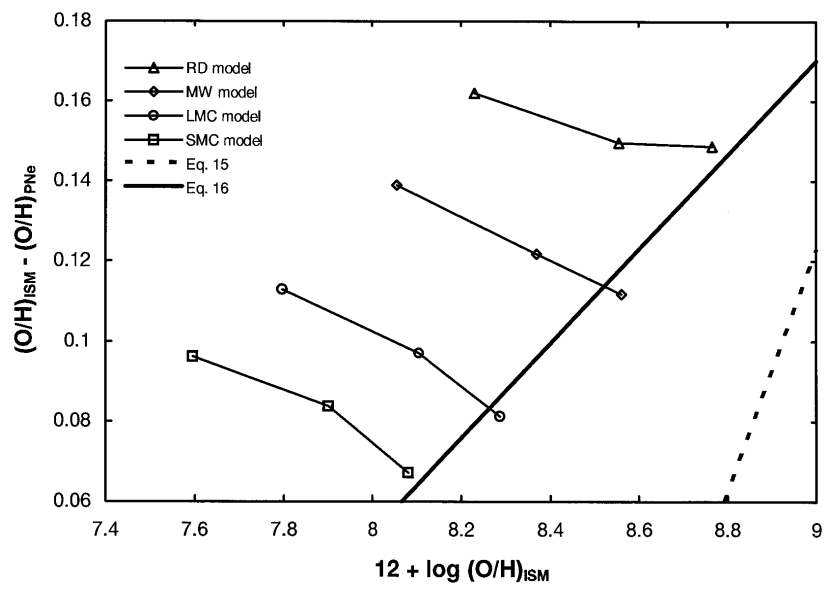

Fig. 6. Here, we plot the abundance gaps for the star-forming models, showing how they evolve with age. For these models, the abundance gap depends upon both the duration and the rate of star formation. The solid line is a straight line fit to the 15 Gyr points (Eq. 16). The dashed line is the fit to the rapid and flat IMF elliptical galaxy models (Eq. 15)

Figure 6 demonstrates that the abundance gap depends upon both the age of the model and the star formation rate. For a constant star formation rate, the rate at which the oxygen abundance changes in the interstellar medium decreases with time. (Adding a fixed amount of newly synthesized oxygen makes a greater change to the interstellar medium abundance if the abundance is initially low). At all times in the star-forming models, the age difference between the youngest and oldest stellar generations that contribute to the population of bright planetary nebulae remains approximately constant. Consequently, the abundance gap decreases with time because the range of ages for the brightest planetary nebulae maps to a smaller range of abundances at older ages. The star formation rate simply exaggerates this effect: stellar generations occupying a fixed range of ages will span a wider range of oxygen abundances as the star formation rate increases.

Given the effect of age in Fig. 6, it is not surprising that the two-burst ellipticals have larger abundance gaps than models that formed their stars in single star-forming episode (see Fig. 5). Not only are later star formation episodes equivalent to stretching out the period of star formation, but they are also equivalent to observing the galaxy at a younger age. As a result, Eq. (15) could perhaps be regarded as a minimum difference between the last epoch oxygen abundance and the mean abundance measured in the brightest planetary nebulae.

Equation (15) may also underestimate the true abundance gap if there exist metallicity-dependent processes that prevent stars from becoming planetary nebulae, e.g., the AGB-manqué evolution. If this were the case, the metal-rich planetary nebulae in our models would be re- moved from the population of bright planetary nebulae, which would increase the abundance gaps.

Presently, the planetary nebulae in the Magellanic Clouds and the Milky Way are the only possible observational connection between real galaxies and Figs. 5 and 6. For the Magellanic Clouds, the relevant data for the HII regions and the planetary nebulae within 1 mag of the PNLF peak are given in Table 2. From these data, the observed abundance gap is $0.02 \pm 0.15$ dex in the LMC and $-0.06 \pm 0.18$ dex in the SMC, whereas Table 4 predicts abundance gaps of $0.08 \pm 0.09 \mathrm{dex}$ and $0.07 \pm 0.09 \mathrm{dex}$ in the LMC and SMC, respectively. Statistically, these observed and predicted abundance gaps are indistinguishable (see Sect. 2.4). For the Milky Way, the mean abundance for the six planetary nebulae within $1 \mathrm{mag}$ of the luminosity function peak in the Méndez et al. (1993) sample is $12+\log (\mathrm{O} / \mathrm{H})=8.63 \pm 0.23$ dex. Using the distances from Méndez et al. (1993) and the observed positions of these objects (e.g., Kaler 1976), their mean galactocentric radius is $7.06 \mathrm{kpc}$ (adopting $7.8 \mathrm{kpc}$ for the solar radius; Feast 1987). Taking the difference in the galactocentric distance scales into account, the observations of $19 \mathrm{H} \mathrm{II}$ regions by Shaver et al. (1983) indicate a mean H II region abundance of $12+\log (\mathrm{O} / \mathrm{H})=8.77 \pm 0.04$ dex at a galactocentric distance of $7.06 \mathrm{kpc}$. A t-test shows that this differs from the planetary nebula mean at the $98.5 \%$ confidence level. For $x_{\mathrm{ISM}}=8.77 \mathrm{dex}$, Eq. (16) predicts an abundance gap of 0.14 dex, identical to that observed.

Since the abundance gap (Eq. 15) is more sensitive to the final interstellar medium oxygen abundance than to the details of how that final oxygen abundance was achieved, it is straightforward to use Eq. (15) to estimate the interstellar medium oxygen abundance when ellipticals stopped forming stars. From observations, one can determine the mean oxygen abundance for planetary nebulae within 1 mag of the PNLF peak. If $x_{\mathrm{PNe}} \gtrsim 8.60$ dex (see Fig. 5), Eq. (15) can be solved for the oxygen abundance in the interstellar medium. If $x_{\mathrm{PNe}}<8.60 \mathrm{dex}$, the abundance gap will depend upon the star formation history, but will be less than $\sim 0.1$ dex, based upon the star-forming series, so it is probably safer to make no correction in this case.

\section{The relationship between diffuse ellipticals and dwarf irregulars}

RM95 used the mean oxygen abundances for the planetary nebulae in NGC 185, NGC 205, and Fornax to investigate whether diffuse elliptical galaxies are related to dwarf irregulars by evolution. If dwarf irregulars evolve to become diffuse ellipticals, star formation in dwarf irregulars must cease, requiring dwarf irregulars to fade in the process. Consequently, if they are the faded remnants of dwarf irregulars, diffuse ellipticals must have higher oxygen abundances than similarly luminous dwarf irregulars. RM95 found that diffuse ellipticals did have 
systematically higher oxygen abundances than similarly luminous dwarf irregulars, but that diffuse ellipticals also had higher $[\mathrm{O} / \mathrm{Fe}]$ ratios than dwarf irregulars of the same oxygen abundance. While the oxygen abundances alone allow that dwarf irregulars fade to become diffuse ellipticals, the $[\mathrm{O} / \mathrm{Fe}]$ ratios indicate that this is unlikely. Since oxygen is produced by type II supernovae, the interstellar medium is enriched in oxygen much more rapidly than it is in iron, to whose production much longer-lived type Ia supernovae contribute significantly. Thus, the $[\mathrm{O} / \mathrm{Fe}]$ ratio is a measure of the rate at which a galaxy consumes its gas. The higher $[\mathrm{O} / \mathrm{Fe}]$ ratios in diffuse ellipticals indicate that they consumed their gas more rapidly than dwarf irregulars. Here, we consider the effect of abundance gaps on these conclusions.

The mean oxygen abundances for the planetary nebula populations in NGC 185, NGC 205, and Fornax studied by RM95 are listed in Table 5. Since these mean abundances are for all planetary nebulae up to 2 mag below the PNLF peak, we computed abundance gaps using the numbers and abundances for planetary nebulae up to 2 mag below the PNLF peak for the $\mathrm{R} X$ and $\mathrm{F} X$ series in Table 4 :

$$
\begin{aligned}
\left(x_{\mathrm{ISM}}-x_{\mathrm{PNe}}\right)_{2 \mathrm{mag}}= & (0.234 \pm 0.024) x_{\mathrm{ISM}} \\
& +(-1.88 \pm 0.22) .
\end{aligned}
$$

The oxygen abundances in the interstellar medium for NGC 185 and NGC 205 in Table 5 were obtained by solving Eq. (17), given the adopted mean oxygen abundances for the planetary nebulae. Since there is only one known planetary nebula in Fornax, we chose not to make an abundance gap correction. The interstellar medium oxygen abundances in Table 5 are those predicted to persist when star formation stopped. In Fig. 7, we compare the predicted last epoch abundances for NGC 185, NGC 205, and Fornax with the metallicity-luminosity relation for dwarf irregulars presented by RM95. Including the effect of the abundance gap for the diffuse ellipticals increases the discrepancy between their oxygen abundances and those of similarly luminous dwarf irregulars.

Table 5. Mean oxygen abundances

\begin{tabular}{lccc}
\hline \hline & NGC 205 & NGC 185 & Fornax \\
\hline Number of PNe $_{\text {Estimated PN mean }}^{\mathrm{a}}$ & 9 & 2 or 3 & 1 \\
Adopted ISM Abundance $^{\mathrm{b}}$ & 8.60 & 8.20 & 7.98 \\
\hline
\end{tabular}

${ }^{\text {a }}$ for planetary nebulae within 2 mag of the PNLF peak

b based upon the estimated PN mean and Eq. (17) for NGC 185 and NGC 205.

Table 6 presents our revised $[\mathrm{O} / \mathrm{Fe}]$ ratios for the diffuse ellipticals. Table 6 is identical to Table 6 of RM95

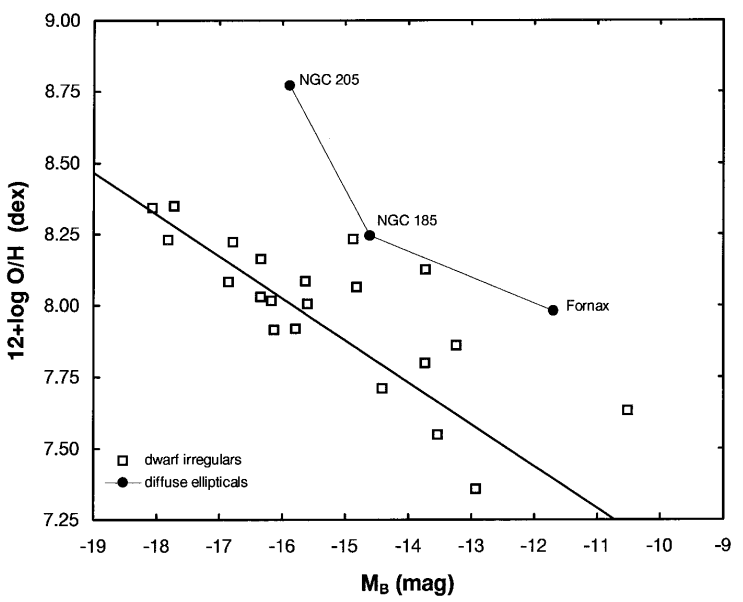

Fig. 7. The metallicity-luminosity relations for dwarf galaxies. The oxygen abundances plotted for the diffuse ellipticals include a correction for the abundance gap (Eq. 17). The line is a linear least squares fit to the dwarf irregulars with $M_{\mathrm{B}}<-15 \mathrm{mag}$ (see RM95)

except for the oxygen abundances adopted for the diffuse ellipticals, which are the last epoch abundances from Table 5. RM95 present an extensive discussion of the derivation of the $[\mathrm{O} / \mathrm{Fe}]$ ratios for the diffuse ellipticals in Table 6 , to which we refer the reader for details. Briefly, the first set of $[\mathrm{O} / \mathrm{Fe}]$ ratios is based upon the maximum $[\mathrm{Fe} / \mathrm{H}]$ and $[\mathrm{O} / \mathrm{H}]$ ratios for the stellar population, while the second set is based upon the mean $[\mathrm{Fe} / \mathrm{H}]$ and $[\mathrm{O} / \mathrm{H}]$ ratios. We derived the first set of $[\mathrm{O} / \mathrm{Fe}]$ ratios by modifying the mean iron abundances using the $[\mathrm{Fe} / \mathrm{H}]$ dispersions, whereas we derived the second set by modifying the oxygen abundances using chemical evolution models. The sources of error are discussed in RM95. In Fig. 8, we plot the $[\mathrm{O} / \mathrm{Fe}]$ ratios for the galaxies as a function of the oxygen abundance in the interstellar medium. The diffuse ellipticals still have systematically higher $[\mathrm{O} / \mathrm{Fe}]$ ratios than dwarf irregulars.

In summary, including the effect of the abundance gap in estimating the interstellar medium oxygen abundances in diffuse ellipticals strengthens the conclusions of RM95. When star formation stopped in diffuse ellipticals, their interstellar medium oxygen abundances were systematically higher than those observed today in similarly luminous dwarf irregulars, and their $[\mathrm{O} / \mathrm{Fe}]$ ratios were systematically higher than those observed in dwarf irregulars with the same oxygen abundance. The simplest explanation for both of these observations is that diffuse ellipticals formed their stars on shorter time scales than dwarf irregulars. Therefore, given their different star formation histories, diffuse ellipticals cannot be the faded remnants of dwarf irregulars. 
Table 6. $[\mathrm{O} / \mathrm{Fe}]$ in diffuse ellipticals

\begin{tabular}{|c|c|c|c|c|c|c|c|c|}
\hline Galaxy & $\begin{array}{c}12+\log (\mathrm{O} / \mathrm{H}) \\
\text { (adopted) }\end{array}$ & mean & $\begin{array}{c}{[\mathrm{Fe} / \mathrm{H}]^{\mathrm{a}}(\mathrm{dex})} \\
\text { maximum }\end{array}$ & Source & $\mu_{1} \mathrm{~b}$ & $Z / Z_{1}{ }^{\mathrm{c}}$ & {$[\mathrm{O} / \mathrm{H}]^{\mathrm{a}}$} & {$[\mathrm{O} / \mathrm{Fe}]^{\mathrm{a}}$} \\
\hline Fornax & 7.98 & -1.40 & -0.70 & 1 & & & -0.95 & $-0.25 \pm 0.18$ \\
\hline NGC 185 & 8.25 & -1.23 & -0.90 & 2 & & & -0.68 & $+0.22 \pm 0.19$ \\
\hline NGC 205 & 8.77 & -0.85 & -0.35 & 3 & & & -0.16 & $+0.19 \pm 0.22$ \\
\hline Fornax & 7.98 & -1.40 & & 1 & 0.77 & 0.47 & -1.28 & $+0.12 \pm 0.18$ \\
\hline NGC 185 & 8.25 & -1.23 & & 2 & 0.61 & 0.44 & -1.04 & $+0.19 \pm 0.20$ \\
\hline NGC 205 & 8.77 & -0.85 & & 3 & 0.19 & 0.31 & -0.66 & $+0.19 \pm 0.30$ \\
\hline Milky Way halo & 8.38 & $\sim-1$ & & 4 & & & & $+0.45 \pm 0.10$ \\
\hline $\mathrm{LMC}$ & 8.35 & -0.30 & & 5 & & & -0.58 & $-0.28 \pm 0.15$ \\
\hline $\mathrm{SMC}$ & 8.03 & -0.69 & & 5 & & & -0.90 & $-0.21 \pm 0.16$ \\
\hline
\end{tabular}

a notation: $[A / B]=\log (n(A) / n(B))-\log (n(A) / n(B)) \odot, n(X)$ being the number density of element $X$.

$\mathrm{b}$ The gas mass fraction when star formation stopped. Calculated using an oxygen yield $y=0.004$ (see discussion and Eq. (6) of RM95).

c The ratio of the mean stellar oxygen abundance to that when star formation stopped, based upon Eq. (5) of RM95. [Fe/H] Sources: 1) Buonanno et al. (1985); 2) Lee et al. (1993); 3) Mould et al. (1984); 4) Tomkin et al. (1992) (for [O/Fe] as well); 5) Russell \& Dopita (1992) (for the oxygen abundances as well).

\section{Conclusions}

This work indicates that bright planetary nebulae are excellent tools for probing the chemical evolution of galaxies. This conclusion is based upon previous studies of starforming galaxies (Richer 1993), where a direct comparison with the interstellar medium is possible, and the predicted properties of planetary nebula populations in galaxies where star formation has ceased.

Although the comparisons of the abundances in planetary nebulae and the interstellar medium for star-forming galaxies are encouraging, it is in galaxies where star formation has stopped that planetary nebulae have their greatest value as abundance probes. To investigate whether, in galaxies lacking star formation, planetary nebulae remain faithful probes of the interstellar medium abundances that persisted when star formation stopped, a code was developed to model the planetary nebula populations in galaxies. This code generates planetary nebula populations based upon the host galaxy's history of star formation, which itself is based upon a self consistent galaxy modelling code (Arimoto \& Yoshii 1986). The models were evaluated by comparing their predicted planetary nebula luminosity functions with those observed in galaxies both with and without star formation, and by comparing the mean densities and oxygen abundances they predicted for bright planetary nebulae in the LMC with those observed. To simultaneously satisfy these requirements, it was necessary to couple the evolution of the nebular shell and the central star, and to impose a relation between the central star mass and the nebular covering factor. Our final models reproduce the first 4 mag of the PNLFs observed in galaxies of different types, as well as the mean densi- ties and abundances observed for planetary nebulae in the LMC (for the LMC model).

Given these successes, the models were used to investigate how well the oxygen abundances in bright planetary nebulae probe the last epoch interstellar medium abundances in galaxies where star formation has long since ceased. The models predict that a gap develops between the abundances observed in bright planetary nebulae and those that existed in the interstellar medium when star formation stopped. The magnitude of this gap increases as the oxygen abundance in the interstellar medium increases. Extending the duration of the star-forming epoch beyond the 1 Gyr that we adopted for our simplest models also increases the size of the abundance gap. The abundance gap is unaffected by changes to the yield of oxygen in the model, so these results are not affected by the slope for the initial mass function. The maximum size of the abundance gap is modest, attaining only $0.35 \mathrm{dex}$ at an interstellar medium oxygen abundance of $12+\log (\mathrm{O} / \mathrm{H})=9.75 \operatorname{dex}(6.6$ times the solar value $)$. Thus, the models predict that, even in galaxies where star formation stopped long ago, bright planetary nebulae should be reliable probes of the oxygen abundance that persisted in the interstellar medium when star formation stopped.

The predicted abundance gaps were compared with those observed in the Magellanic Clouds and the disk of the Milky Way. In the Magellanic Clouds, the observed and predicted abundance gaps are statistically compatible. For the Milky Way disk, models predict an abundance gap of $0.14 \mathrm{dex}$, which is identical to the value observed.

In light of these results, we re-analyzed the oxygen abundances for the planetary nebulae in NGC 185, NGC 205, and Fornax from RM95 to investigate whether 


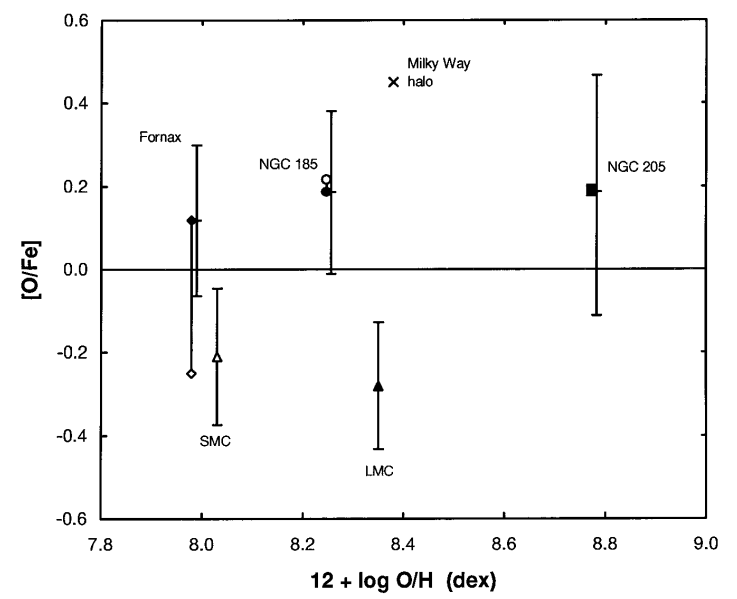

Fig. 8. Comparison of the $[\mathrm{O} / \mathrm{Fe}]$ ratios for diffuse ellipticals with those for the Magellanic Clouds and the Milky Way halo. For the diffuse ellipticals, the filled symbols denote the $[\mathrm{O} / \mathrm{Fe}]$ ratios computed from the closed box model of chemical evolution, while the open symbols denote $[\mathrm{O} / \mathrm{Fe}]$ ratios derived from the maximum iron abundance. (For NGC 205, the $[\mathrm{O} / \mathrm{Fe}]$ ratios from both methods are identical). The horizontal line indicates the solar $[\mathrm{O} / \mathrm{Fe}]$ ratio. The error bars include the uncertainties in the oxygen and iron abundances, and, for the diffuse ellipticals, the effect of allowing the yield of oxygen to vary between 0.002 and 0.02 . For clarity, the error bars for the diffuse ellipticals are shifted slightly to the right of the closed box $[\mathrm{O} / \mathrm{Fe}]$ ratios

diffuse ellipticals could be related to dwarf irregulars by evolution. Accounting for the abundance gap only served to exaggerate the differences found by RM95: when star formation stops, diffuse ellipticals have systematically higher oxygen abundances than equally luminous dwarf irregulars, and higher $[\mathrm{O} / \mathrm{Fe}]$ ratios than dwarf irregulars with similar oxygen abundances. The simplest explanation for both of these trends is that diffuse ellipticals consume their gas more rapidly than dwarf irregulars before expelling or losing what remains of it. Consequently, diffuse ellipticals cannot be the faded remnants of dwarf irregulars, for the star formation histories in these two types of galaxies are fundamentally different.

Acknowledgements. MGR would like to thank Dr. Emanuel Vassiliadis for making his evolutionary tracks for planetary nebula central stars available well before publication, and Dr. Robin Ciardullo for communicating important results prior to publication. MGR would also like to acknowledge the Ontario Ministry of Colleges and Universities, Marshall McCall, and the Natural Sciences and Engineering Research Council of Canada for their financial support. MLM thanks the Natural Sciences and Engineering Research Council of Canada for its continuing support. This work was financially supported in part by Grant-in-Aid (Nos. 06640349, 072222206) for the Scientific Research of the Japanese Ministry of Education, Culture, Sport, and Science.

\section{References}

Allen C.W., 1973, Astrophys. Quant., London: Athlone

Arimoto N., Yoshii Y., 1986, A\&A 164, 260

Arimoto N., Yoshii Y., Takahara F., 1992, A\&A 253, 21

Boesgaard A.M., 1989, ApJ 336, 798

Boroson T.A., Liebert J., 1989, ApJ 339, 844

Bressan A., Fagotto F., Bertelli G., Chiosi C., 1993, A\&AS 100, 647

Buonanno R., Corsi C.E., Fusi Pecci F., Hardy E., Zinn R., 1985, A\&A 152, 65

Ciardullo R., 1995, in IAU Highlights of Astronomy Vol. 10, Appenzeller I. (ed.). Dordrecht: Kluwer, p. 507

Ciardullo R., Jacoby G.H., Ford H.C., 1989a, ApJ 344, 715

Ciardullo R., Jacoby G.H., Harris W.E., 1991, ApJ 383, 487

Ciardullo R., Jacoby G.H., Ford H.C., Neill J.D., 1989b, ApJ 339,53

Dopita M.A., Meatheringham S.J., 1990, ApJ 357, 140

Dopita M.A., Meatheringham S.J., 1991a, ApJ 367, 115

Dopita M.A., Meatheringham S.J., 1991b, ApJ 377, 480

Dopita M.A., Jacoby G.H., Vassiliadis E., 1992, ApJ 389, 27

Dopita M.A., Meatheringham S.J., Webster B.L., Ford H.C., 1988, ApJ 327, 639

Fagotto F., Bressan A., Bertelli G., Chiosi C., 1994a, A\&AS 104, 365

Fagotto F., Bressan A., Bertelli G., Chiosi C., 1994b, A\&AS 105,29

Feast M.W., 1987, in The Galaxy, Gilmore G. \& Carswell B. (ed.). Dordrecht: Reidel, p. 1

Gabler R., Kudritzki R.P., Méndez R.H., 1991, A\&A 245, 587

Henize K.G., Westerlund B.E., 1963, ApJ 137, 747

Huchra J., Stauffer J., van Speybroeck L., 1982, ApJ 259, L57

Hui X., Ford H.C., Ciardullo R., Jacoby G.H., 1993, ApJ 414, 463

Jacoby G.H., 1980, ApJS 42, 1

Jacoby G.H., 1989, ApJ 339, 39

Jacoby G.H., Ford H.C., 1986, ApJ 304, 490

Jacoby G.H., Kaler J.B., 1993, ApJ 417, 209

Jacoby G.H., Walker A.R., Ciardullo R., 1990, ApJ 365, 471

Johnson J.J., Jones T.J., 1991, AJ 101, 1735

Kaler J.B., 1976, ApJS 31, 517

Kaler J.B., Jacoby G.H., 1991, ApJ 372, 215

Lee M. G., Freedman W.L., Madore B.F., 1993, ApJ 417, 553

Lequeux J., 1984, in Structure and Evolution of the Magellanic Clouds, van den Bergh S. \& de Boer K.S. (eds.). Dordrecht: Reidel, p. 67

Maeder A., 1992, A\&A 264, 105

McCarthy J.K., Mould J.R., Méndez R.H., Kudritzki R.P., Husfeld D., Herrero A., Groth H.G., 1990, ApJ 351, 230

McMillan R., Ciardullo R., Jacoby G.H., 1993, ApJ 416, 62

Méndez R.H., Kudritzki R.P., Herrero A., 1992, A\&A 260, 329

Méndez R.H., Kudritzki R.P., Ciardullo R., Jacoby G.H., 1993, A\&A 275,534

Mould J., Kristian J., Da Costa G.S., 1984, ApJ 278, 575

Osterbrock D.E., 1989, Astrophysics of Gaseous Nebulae and Active Galactic Nuclei, Mill Valley: University Science Books

Peimbert M., 1990, Rep. Prog. Phys. 53, 1559

Rana N.C., 1991, ARA\&A 29, 129

Renzini A., Buzzoni A., 1986, in Spectral Evolution of Galaxies, Chiosi C. \& Renzini A. (eds.). Dordrecht: Reidel, p. 195 
Richer M.G., 1993, ApJ 415, 240

Richer M.G., 1994, PhD thesis, York University

Richer M.G., McCall M.L., 1995, ApJ 445, 642 (RM95)

Russell S.C., Dopita M.A., 1992, ApJ 384, 508

Salpeter E.E., 1955, ApJ 121, 161

Schönberner D., 1987, in Planetary Nebulae: IAU Symposium 131, Torres-Peimbert S. (ed.). Dordrecht: Kluwer, p. 463

Shaver P.A., McGee R.X., Newton L.M., Danks A.C., Pottasch S.R., 1983, MNRAS 204, 53

Tomkin J., Lemke M., Lambert D.L., Sneden C., 1992, AJ 104, 1568
Trammell S.R., Dinerstein H.L., Goodrich R.W., 1994, AJ 108, 984

van den Bergh S., 1969, ApJS 19, 145

Vassiliadis E., Wood P.R., 1994, ApJS 92, 125

Vassiliadis E., Dopita M.A., Morgan D.H., Bell J.F., 1992, ApJS 83, 87

Weidemann V., 1987, A\&A 188, 74

Weidemann V., 1990, ARA\&A 28, 103 